\title{
THE OPERATOR SYSTEM OF TOEPLITZ MATRICES
}

\author{
DOUGLAS FARENICK
}

\begin{abstract}
A recent paper of A. Connes and W.D. van Suijlekom [Comm. Math. Phys. 383 (2021), pp. 2021-2067] identifies the operator system of $n \times n$ Toeplitz matrices with the dual of the space of all trigonometric polynomials of degree less than $n$. The present paper examines this identification in somewhat more detail by showing explicitly that the Connes-van Suijlekom isomorphism is a unital complete order isomorphism of operator systems. Applications include two special results in matrix analysis: (i) that every positive linear map of the $n \times n$ complex matrices is completely positive when restricted to the operator subsystem of Toeplitz matrices and (ii) that every linear unital isometry of the $n \times n$ Toeplitz matrices into the algebra of all $n \times n$ complex matrices is a unitary similarity transformation.

An operator systems approach to Toeplitz matrices yields new insights into the positivity of block Toeplitz matrices, which are viewed herein as elements of tensor product spaces of an arbitrary operator system with the operator system of $n \times n$ complex Toeplitz matrices. In particular, it is shown that min and max positivity are distinct if the blocks themselves are Toeplitz matrices, and that the maximally entangled Toeplitz matrix $\xi_{n}$ generates an extremal ray in the cone of all continuous $n \times n$ Toeplitz-matrix valued functions $f$ on the unit circle $S^{1}$ whose Fourier coefficients $\hat{f}(k)$ vanish for $|k| \geq n$. Lastly, it is noted that all positive Toeplitz matrices over nuclear $C^{*}$-algebras are approximately separable.
\end{abstract}

\section{INTRODUCTION}

Toeplitz operators and matrices are among the most intensively studied and best understood of all classes of Hilbert space operators; in this paper, they are considered from the perspective of the unital selfadjoint linear subspaces they generate. These subspaces of matrices and operators are concrete instances of operator systems, which in the abstract refer to matrix-ordered involutive complex vector spaces possessing an Archimedean order unit [8].

In addition to classical Toeplitz matrices, this paper considers block Toeplitz matrices, which are matrices $x$ of the form

$$
x=\left[\begin{array}{cccccc}
s_{0} & s_{-1} & s_{-2} & \ldots & s_{-n+2} & s_{-n+1} \\
s_{1} & s_{0} & s_{-1} & s_{-2} & \ldots & s_{-n+2} \\
s_{2} & s_{1} & s_{0} & s_{-1} & \ddots & \vdots \\
\vdots & \ddots & \ddots & \ddots & \ddots & s_{-2} \\
s_{n-2} & & \ddots & \ddots & \ddots & s_{-1} \\
s_{n-1} & s_{n-2} & \ldots & s_{2} & s_{1} & s_{0}
\end{array}\right],
$$

Received by the editors April 3, 2021, and, in revised form, May 22, 2021, and June 7, 2021. 2020 Mathematics Subject Classification. Primary 46L07, 47L05.

This work was supported in part by the NSERC Discovery Grant program. 
for some $s_{-n+1}, \ldots, s_{n-1}$ in an operator system $\mathcal{S}$. The present paper considers such matrices $x$ when $\mathcal{S}$ is an operator system, and addresses the issue of positivity for these Toeplitz matrices, particularly in the cases $\mathcal{S}=\mathcal{M}_{m}(\mathbb{C})$, the $\mathrm{C}^{*}$-algebra of $m \times m$ complex matrices, and $\mathcal{S}=C\left(S^{1}\right)^{(m)}$, the operator system of $m \times m$ complex Toeplitz matrices.

The work in this paper is strongly motivated by recent results of Connes and van Suijlekom [9] which, among other things, identify the operator system of Toeplitz matrices with the dual space of a function system of trigonometric polynomials. To explain the contributions of the present paper and set the notation, let $C\left(S^{1}\right)$ denote the unital abelian $\mathrm{C}^{*}$-algebra of all continuous functions $f: S^{1} \rightarrow \mathbb{C}$, where $S^{1} \subset \mathbb{C}$ is the unit circle. For each $n \in \mathbb{N}$, let $C\left(S^{1}\right)_{(n)}$ denote the vector space of those $f \in C\left(S^{1}\right)$ for which the Fourier coefficients $\hat{f}(k)$ of $f$ satisfy $\hat{f}(k)=0$ for every $k \in \mathbb{Z}$ such that $|k| \geq n$. Thus, every $f \in C\left(S^{1}\right)_{(n)}$ is given by

$$
f(z)=\sum_{k=-n+1}^{n-1} \alpha_{k} z^{k},
$$

as a function of $z \in S^{1}$, where each $\alpha_{k}=\frac{1}{2 \pi} \int_{0}^{2 \pi} f\left(e^{i \theta}\right) e^{-i k \theta} d \theta$. The vector space $C\left(S^{1}\right)_{(n)}$ is an operator system via the matrix ordering that arises from the identification of $\mathcal{M}_{p}\left(C\left(S^{1}\right)_{(n)}\right)$, the space of $p \times p$ matrices with entries from $C\left(S^{1}\right)_{(n)}$, with the space of continuous functions $F: S^{1} \rightarrow \mathcal{M}_{p}(\mathbb{C})$, and where the Archimedean order unit is the canonical one (namely, the constant function $\chi_{0}: S^{1} \rightarrow \mathbb{C}$ given by $\chi_{0}(z)=1$, for $\left.z \in S^{1}\right)$.

The operator system of all $n \times n$ Toeplitz matrices over $\mathbb{C}$ is denoted by $C\left(S^{1}\right)^{(n)}$; the identity matrix in $\mathcal{M}_{n}(\mathbb{C})$ is the canonical Archimedean order unit for $C\left(S^{1}\right)^{(n)}$.

As explained in 8 , if $\mathcal{R}$ is an operator system and $\mathcal{R}^{d}$ denotes its dual space, then $\mathcal{R}^{d}$ is a matrix-ordered $*$-vector space. Specifically, a matrix $\Phi=\left[\varphi_{i j}\right]_{i, j=1}^{p}$ of linear functionals $\varphi_{i j}: \mathcal{R} \rightarrow \mathbb{C}$ is considered positive whenever the linear map $r \mapsto\left[\varphi_{i j}(r)\right]_{i, j=1}^{p}$ is a completely positive linear map of $\mathcal{R}$ into the algebra $\mathcal{M}_{p}(\mathbb{C})$. Furthermore, if $\phi: \mathcal{R} \rightarrow \mathcal{S}$ is a linear map of operator systems and $\phi^{d}: \mathcal{S}^{d} \rightarrow \mathcal{R}^{d}$ denotes the adjoint transformation as linear mapping of matrix-ordered $*$-vector spaces, then $\phi$ is positive if and only if $\phi^{d}$ is positive. Likewise, $\phi \otimes \mathrm{id}_{\mathcal{M}_{p}(\mathbb{C})}$ is positive if and only if $\phi^{d} \otimes \operatorname{id}_{\mathcal{M}_{p}(\mathbb{C})}$ is positive, for $p \in \mathbb{N}$.

If an operator system $\mathcal{R}$ has finite dimension, then any faithful state $\varphi$ on $\mathcal{R}$ serves an Archimedean order unit for the matrix-ordered $*$-vector space $\mathcal{R}^{d}$, thereby giving $\mathcal{R}^{d}$ the structure of an operator system. Because the linear functional $\mathfrak{e}_{(n)}$ : $C\left(S^{1}\right)_{(n)} \rightarrow \mathbb{C}$ given by

$$
\mathfrak{e}_{(n)}[f]=\hat{f}(0)=\frac{1}{2 \pi} \int_{0}^{2 \pi} f\left(e^{i \theta}\right) d \theta
$$

is a faithful state, we shall henceforth designate $\mathfrak{e}_{(n)}$ as the Archimedean order unit for the operator system dual of $C\left(S^{1}\right)_{(n)}$.

The category $\mathcal{O}_{1}$ has as its objects operator systems, and as its morphisms unital completely positive linear maps. Therefore, an isomorphism in this category is a unital completely positive linear map $\phi: \mathcal{R} \rightarrow \mathcal{S}$ between operator systems $\mathcal{R}$ and $\mathcal{S}$ such that $\phi$ is a linear bijection and both $\phi$ and $\phi^{-1}$ are completely positive. (The complete positivity of a linear bijection $\phi$ is not sufficient to imply the complete positivity of its inverse $\phi^{-1}$.) Such a linear isomorphism is called a unital complete 
order isomorphism and we denote the existence of such an isomorphism between operator systems $\mathcal{R}$ and $\mathcal{S}$ with the notation

$$
\mathcal{R} \simeq \mathcal{S}
$$

This notation above has its own ambiguity, as explicit reference to the Archimedean order units of $\mathcal{R}$ and $\mathcal{S}$ is not made. In this paper, whenever we are speaking of specific operator systems $\mathcal{R}$ and $\mathcal{S}$ that have had Archimedean order units $e_{\mathcal{R}}$ and $e_{\mathcal{S}}$ explicitly designated, then $\mathcal{R} \simeq \mathcal{S}$ implies that there is a complete order isomorphism between these operator systems that sends $e_{\mathcal{R}}$ to $e_{\mathcal{S}}$.

The main result of this paper is the following isomorphism theorem in the operator system category; the remaining results in this paper are derived from this isomorphism.

Theorem 1.1. $C\left(S^{1}\right)^{(n)} \simeq\left(C\left(S^{1}\right)_{(n)}\right)^{d}$ for every $n \in \mathbb{N}$.

Theorem 1.1, as stated above, was proved in [14, Theorem 4.5] for $n=2$ using an approach rather different from the approach of the present paper. In that paper, the operator system $C\left(S^{1}\right)_{(2)}$ arises as $\mathcal{S}_{1}$, the operator system generated by a universal unitary operator. The approach in the present paper is inspired by and based upon an elegant argument of Connes and van Suijlekom [9, Proposition 4.6], which proves that there is a linear unital order isomorphism $\phi$ between the operator system $C\left(S^{1}\right)^{(n)}$ and the operator system dual $\left(C\left(S^{1}\right)_{(n)}\right)^{d}$. The new contribution in Theorem 1.1 is the proof that this linear unital order isomorphism $\phi$ is a complete order isomorphism.

Theorem 1.1 has a curious consequence for positive linear maps $\psi: \mathcal{M}_{n}(\mathbb{C}) \rightarrow$ $\mathcal{M}_{m}(\mathbb{C})$. Recall that a positive linear map $\psi: \mathcal{M}_{n}(\mathbb{C}) \rightarrow \mathcal{M}_{m}(\mathbb{C})$ is decomposable if it is the sum of completely positive and completely co-positive linear maps; that is, if there are $n \times m$ matrices $a_{1}, \ldots, a_{k}$ and $b_{1}, \ldots, b_{\ell}$ such that

$$
\psi(x)=\sum_{i=1}^{k} a_{i}^{*} x a_{i}+\sum_{j=1}^{\ell} b_{j}^{*} x^{t} b_{j},
$$

where $x^{t}$ denotes the transpose map on $\mathcal{M}_{n}(\mathbb{C})$ (which is positive but not completely positive). If $x$ is a Toeplitz matrix, then there is a unitary $u$ independent of $x$ for which $x^{t}=u^{*} x u$ (namely, $u=\sum_{i=1}^{n} e_{i, n-i+1}$, where $e_{i j}$ is a matrix unit of $\mathcal{M}_{n}(\mathbb{C})$ ). Thus, the restriction of a decomposable positive linear map to the Toeplitz operator system $C\left(S^{1}\right)^{(n)}$ is completely positive. However, for all $n \geq 3$ there exist indecomposable positive linear maps on $\mathcal{M}_{n}(\mathbb{C})[26$, which makes the following assertion somewhat unexpected.

Theorem 1.2. The restriction of every positive linear map $\psi: \mathcal{M}_{n}(\mathbb{C}) \rightarrow \mathcal{M}_{m}(\mathbb{C})$ to the operator system $C\left(S^{1}\right)^{(n)}$ of Toeplitz matrices is completely positive.

Theorems 1.1 and 1.2 also lead to a new proof of the following theorem from 13 . concerning linear isometries of operator systems of Toeplitz matrices.

Theorem 1.3. If $\phi: C\left(S^{1}\right)^{(n)} \rightarrow \mathcal{M}_{n}(\mathbb{C})$ is a unital linear isometry, then there exists a unitary matrix $v$ such that $\phi(x)=v^{*} x v$, for every $x \in C\left(S^{1}\right)^{(n)}$.

In 6. to address questions of matrix positivity for Toeplitz matrices $x$ over operator systems $\mathcal{S}$, as in equation (1.1), operator system tensor products will have a role in giving meaning to various notions of positivity. All of these notions 
coincide if the $s_{k}$ are assumed to be elements of $\mathcal{M}_{m}(\mathbb{C})$ (or, indeed, of any nuclear $\mathrm{C}^{*}$-algebra), but the notions become distinct if the elements $s_{k}$ in the Toeplitz matrix $x$ are themselves Toeplitz matrices (Corollary 6.6).

Lastly, there is a well-known relationship between Toeplitz operators on the Hardy space of $S^{1}$ and essentially-bounded functions (symbols) on $S^{1}$; this relationship is addressed in $₫ 4$ for the symbol spaces $C\left(S^{1}\right)_{(n)}$, giving rise to the identification in the operator system category of certain Toeplitz operators as dual elements to finite Toeplitz matrices.

\section{Preliminaries}

2.1. Terminology. Throughout this paper, when referring to a positive matrix or operator, what is meant is a selfadjoint operator with spectrum contained in the halfline $[0, \infty)$; thus, "positive" is the same as "positive semidefinite" in this terminology.

The Schur-Hadamard product (or entry-wise product) of $d \times d$ matrices $a$ and $b$ is denoted by $a \circ b$ and is the matrix in which each $(i, j)$-entry of $a \circ b$ is given by the product of the corresponding $(i, j)$-entries of $a$ and $b$. The Schur-Hadamard isometry is the linear map $v: \mathbb{C}^{d} \rightarrow \mathbb{C}^{d} \otimes \mathbb{C}^{d}$ that sends the $k$-th canonical orthonormal basis vector $e_{k}$ of $\mathbb{C}^{d}$ to $e_{k} \otimes e_{k} \in \mathbb{C}^{d} \otimes \mathbb{C}^{d}$; this isometry has the property that $a \circ b=v^{*}(a \otimes b) v$, implying that $a \otimes b$ is positive if $a$ and $b$ are positive.

The canonical matrix units for a full matrix algebra $\mathcal{M}_{n}(\mathbb{C})$ shall be denoted, henceforth, by $e_{i j}$, and the analytic shift matrix in $\mathcal{M}_{n}(\mathbb{C})$ is the lower-triangular Toeplitz matrix $s$ given

$$
s=\sum_{j=1}^{n-1} e_{j+1, j}=\left[\begin{array}{ccccc}
0 & & & & \\
1 & 0 & & & \\
& 1 & 0 & & \\
& & \ddots & \ddots & \\
& & & 1 & 0
\end{array}\right] .
$$

The algebra of bounded linear operators acting on a Hilbert space $\mathcal{H}$ is denoted by $\mathcal{B}(\mathcal{H})$. An operator $x \in \mathcal{B}(\mathcal{H})$ is irreducible if the commutant of $\left\{x, x^{*}\right\}$ is trivial (i.e., consists only of scalar multiples of the identity operator). Likewise, an operator subsystem $\mathcal{S} \subseteq \mathcal{B}(\mathcal{H})$ is irreducible if $\mathcal{S}^{\prime}$ (the commutant of $\mathcal{S}$ ) is trivial. Thus, the analytic Toeplitz matrix $s$ is irreducible, along with any operator subsystem of $\mathcal{M}_{n}(\mathbb{C})$ that contains $s$.

2.2. Operator systems. Formally, an operator system is a triple $\left(\mathcal{S},\left\{\mathcal{C}_{n}\right\}_{n \in \mathbb{N}}, e_{\mathcal{S}}\right)$ consisting of:

(1) a complex $*$-vector space $\mathcal{S}$;

(2) a family $\left\{\mathcal{C}_{n}\right\}_{n \in \mathbb{N}}$ of proper cones $\mathcal{C}_{n} \subseteq \mathcal{M}_{n}(\mathcal{S})_{\text {sa }}$ of selfadjoint matrices with the properties that $\mathcal{C}_{n} \oplus \mathcal{C}_{m} \subseteq \mathcal{C}_{n+m}$ and $\gamma^{*} \mathcal{C}_{n} \gamma \subseteq \mathcal{C}_{m}$ for all $n, m \in \mathbb{N}$ and all linear transformations $\gamma: \mathbb{C}^{m} \rightarrow \mathbb{C}^{n}$; and

(3) an Archimedean order unit $e_{\mathcal{S}}$ for the ordered real vector space $\mathcal{S}_{\text {sa }}$.

In concrete situations, operator systems arise as unital $*$-closed subspaces of unital $\mathrm{C}^{*}$-algebras.

If $\mathcal{S}$ and $\mathcal{R}$ are operator systems with Archimedean order units $e_{\mathcal{S}}$ and $e_{\mathcal{R}}$, then $\mathcal{S}$ is said to be an operator subsystem of $\mathcal{R}$ if $\mathcal{S} \subseteq \mathcal{R}$ and $e_{\mathcal{S}}=e_{\mathcal{R}}$. A unital completely positive linear map $\phi: \mathcal{S} \rightarrow \mathcal{T}$ is a unital complete order embedding if 
$\phi$ is a unital complete order isomorphism between $\mathcal{S}$ and the operator subsystem $\phi(\mathcal{S})$ of $\mathcal{T}$.

The embedding theorem of Choi and Effros [8] states that every operator system $\mathcal{R}$ is unitally completely order isomorphic to an operator subsystem of $\mathcal{B}(\mathcal{H})$, for some Hilbert space $\mathcal{H}$. Therefore, every operator system $\mathcal{R}$ is capable of generating a $\mathrm{C}^{*}$-algebra. Two such $\mathrm{C}^{*}$-algebras of note are the $\mathrm{C}^{*}$-envelope, $\mathrm{C}_{\mathrm{e}}^{*}(\mathcal{R})$, and the universal $\mathrm{C}^{*}$-algebra, $\mathrm{C}_{\mathrm{u}}^{*}(\mathcal{R})$, which satisfy, respectively, minimal and maximal universal properties 16,24 .

The following result from the literature shows that $\mathrm{C}^{*}$-envelope of an operator system can be nuclear, while the universal $\mathrm{C}^{*}$-algebra of the same operator system need not be exact.

Theorem 2.1. For every $n \geq 2$,

(1) $\mathrm{C}_{\mathrm{e}}^{*}\left(C\left(S^{1}\right)^{(n)}\right)=\mathcal{M}_{n}(\mathbb{C})$,

(2) $\mathrm{C}_{\mathrm{e}}^{*}\left(C\left(S^{1}\right)_{(n)}\right)=C\left(S^{1}\right)$, and

(3) neither $\mathrm{C}_{\mathrm{u}}^{*}\left(C\left(S^{1}\right)^{(n)}\right)$ nor $\mathrm{C}_{\mathrm{u}}^{*}\left(C\left(S^{1}\right)_{(n)}\right)$ is an exact $C^{*}$-algebra.

Proof. The first two assertions are given by Propositions 4.2 and 4.3 of [9], while the third assertion is derived from Proposition 6.3 of [20].

Definition 2.2. An operator subsystem $\mathcal{R}$ of a unital $\mathrm{C}^{*}$-algebra $\mathcal{A}$ is hyperrigid in $\mathcal{A}$ if, for every representation $\pi: \mathcal{A} \rightarrow \mathcal{B}\left(\mathcal{H}_{\pi}\right)$ of $\mathcal{A}$, the ucp map $\pi_{\mid \mathcal{R}}: \mathcal{R} \rightarrow \mathcal{B}\left(\mathcal{H}_{\pi}\right)$ has a unique extension to a completely positive linear map on $\mathcal{A}$.

In the definition above, the unique completely positive extension of the restriction $\pi_{\mid \mathcal{R}}$ of $\pi$ to $\mathcal{R}$ is of course $\pi$ itself.

Proposition 2.3. The operator system $C\left(S^{1}\right)^{(n)}$ is hyperrigid in $M_{n}(\mathbb{C})$, for all $n \geq 2$.

Proof. Write the lower-triangular shift matrix $s \in C\left(S^{1}\right)^{(n)}$ as $s=(1 / 2)(u+w)$, where $u$ and $w$ are the unitary Toeplitz matrices

$$
u=e_{1, n}+\sum_{i=2}^{n} e_{i, i-1} \text { and } w=-e_{1, n}+\sum_{i=2}^{n} e_{i, i-1} .
$$

As the algebra generated by $s$ and $s^{*}$ is $\mathcal{M}_{n}(\mathbb{C})$, this shows that the unitary Toeplitz matrices generate the algebra $\mathcal{M}_{n}(\mathbb{C})$. The hyperrigidity of $C\left(S^{1}\right)^{(n)}$ now follows from [17, Lemma 3.11], which states that if the unitary elements of an operator subsystem $\mathcal{R}$ of a unital $\mathrm{C}^{*}$-algebra $\mathcal{A}$ generate $\mathcal{A}$, then $\mathcal{R}$ is hyperrigid in $\mathcal{A}$.

The following lemmas on automatic complete positivity will be useful.

Lemma 2.4. If $\mathcal{R}$ and $\mathcal{S}$ are operator systems such that the $C^{*}$-envelope of $\mathcal{S}$ is abelian, then every positive linear map $\phi: \mathcal{R} \rightarrow \mathcal{S}$ is completely positive.

Proof. The unital complete order embedding $\iota_{\mathrm{e}}: \mathcal{S} \rightarrow \mathrm{C}_{\mathrm{e}}^{*}(\mathcal{S})$ has the property that, for any $X \in \mathcal{M}_{p}(\mathcal{S})$, the matrix $\iota_{e}^{[p]}(X)$ is positive in $\mathcal{M}_{p}\left(\mathrm{C}_{\mathrm{e}}^{*}(\mathcal{S})\right)$ if and only if $X$ is positive in $\mathcal{M}_{p}(\mathcal{S})$. In other words,

$$
\mathcal{S} \simeq \iota_{\mathrm{e}}(\mathcal{S})
$$

As every positive linear map of an operator system into a unital abelian $\mathrm{C}^{*}$-algebra is completely positive [25, Theorem 3.9], we deduce that $\iota_{\mathrm{e}} \circ \phi$ is completely positive. Hence, $\phi$ is necessarily completely positive. 
Lemma 2.5. If $\mathcal{R}$ is a finite-dimensional operator system such that $\mathrm{C}_{\mathrm{e}}^{*}\left(\mathcal{R}^{d}\right)$ is abelian, then every positive linear map $\phi: \mathcal{R} \rightarrow \mathcal{B}(\mathcal{H})$ is completely positive.

Proof. The range of $\phi$ is a $*$-closed subspace of $\mathcal{B}(\mathcal{H})$. Let $\mathcal{T}$ denote the operator subsystem of $\mathcal{B}(\mathcal{H})$ spanned by $\phi(\mathcal{R})$ and the identity operator on $\mathcal{H}$; thus, $\mathcal{T}$ is a finite-dimensional operator system and $\phi$ is a positive linear map $\mathcal{R} \rightarrow \mathcal{T}$. Consider the dual linear map $\phi^{d}: \mathcal{T}^{d} \rightarrow \mathcal{R}^{d}$; because $\phi$ is positive, the dual map $\phi^{d}$ is positive. Because $\mathrm{C}_{\mathrm{e}}^{*}\left(\mathcal{R}^{d}\right)$ is abelian, Lemma 2.4 implies that $\phi^{d}$ is completely positive; hence, $\left(\phi^{d}\right)^{d}=\phi$ is completely positive.

2.3. Tensor representations. Suppose that an operator system $\mathcal{R}$ has a linear basis $\left\{r_{\ell}\right\}_{\ell}$. Fix $p \in \mathbb{N}$ and let $\left\{e_{i j}\right\}_{i, j=1}^{p}$ denote the canonical basis of $\mathcal{M}_{p}(\mathbb{C})$ given by the standard matrix units. The algebraic tensor product $\mathcal{R} \otimes \mathcal{M}_{p}(\mathbb{C})$ may be identified with the set of $p \times p$ matrices with entries from $\mathcal{R}$ or, alternatively, as " $\mathcal{R}$ with entries from $\mathcal{M}_{p}(\mathbb{C}) . "$ As it is this second viewpoint that is required, a brief explanation of what this phrase means is given below.

If $x \in \mathcal{R} \otimes \mathcal{M}_{p}(\mathbb{C})$, then there exists scalars $\alpha_{\ell i j} \in \mathbb{C}$, finitely many of which are nonzero, such that

$$
x=\sum_{\ell} \sum_{i} \sum_{j} \alpha_{\ell i j}\left(r_{\ell} \otimes e_{i j}\right)=\sum_{i} \sum_{j}\left(\sum_{\ell} \alpha_{\ell i j} r_{\ell}\right) \otimes e_{i j}=\sum_{i} \sum_{j} x_{i j} \otimes e_{i j},
$$

where $x_{i j} \in \mathcal{R}$ is the element $\sum_{\ell} \alpha_{\ell i j} r_{\ell}$. This gives the identification of $\mathcal{R} \otimes \mathcal{M}_{p}(\mathbb{C})$ as $p \times p$ matrices with entries from $\mathcal{R}$, which is the usual point of view in the theory of operator systems and operator spaces. On the other hand, we may express $x \in \mathcal{R} \otimes \mathcal{M}_{p}(\mathbb{C})$ as

$$
x=\sum_{\ell} \sum_{i} \sum_{j} \alpha_{\ell i j}\left(r_{\ell} \otimes e_{i j}\right)=\sum_{\ell} r_{\ell} \otimes\left(\sum_{i} \sum_{j} \alpha_{\ell i j} e_{i j}\right)=\sum_{\ell} r_{\ell} \otimes a_{\ell},
$$

where $a_{\ell} \in \mathcal{M}_{p}(\mathbb{C})$ is the matrix $\sum_{i} \sum_{j} \alpha_{\ell i j} e_{i j}$. This give us the conceptual identification of $\mathcal{R} \otimes \mathcal{M}_{p}(\mathbb{C})$ as $\mathcal{R}$ with entries from $\mathcal{M}_{p}(\mathbb{C})$.

Similarly, if $\phi: \mathcal{R} \rightarrow \mathcal{S}$ is a linear map of operator systems, then the linear map $\phi^{[p]}=\phi \otimes \operatorname{id}_{\mathcal{M}_{p}(\mathbb{C})}: \mathcal{R} \otimes \mathcal{M}_{p}(\mathbb{C}) \rightarrow \mathcal{S} \otimes \mathcal{M}_{p}(\mathbb{C})$ evaluated at $x \in \mathcal{R} \otimes \mathcal{M}_{p}(\mathbb{C})$ is, in our two views of $x$ above, given by

$$
\phi^{[p]}[x]=\phi^{[p]}\left[\sum_{\ell} \sum_{i} \sum_{j} \alpha_{\ell i j}\left(r_{\ell} \otimes e_{i j}\right)\right]=\sum_{i} \sum_{j} \phi\left(x_{i j}\right) \otimes e_{i j}=\sum_{\ell} \phi\left(r_{\ell}\right) \otimes a_{\ell} .
$$

2.4. Canonical linear bases. The canonical linear basis for the operator system $C\left(S^{1}\right)^{(n)}$ of Toeplitz matrices is given by powers of the shift and their adjoints. That is, if $\left\{r_{-n+1}, \ldots, r_{0}, \ldots, r_{n-1}\right\}$ is the set of matrices defined by

$$
r_{k}=\left\{\begin{array}{lll}
s^{k} & : & \text { if } k \geq 0 \\
\left(s^{*}\right)^{k} & : & \text { if } k<0
\end{array}\right\},
$$

then $\left\{r_{-n+1}, \ldots, r_{0}, \ldots, r_{n-1}\right\}$ is a linear basis of $C\left(S^{1}\right)^{(n)}$. The identity matrix $r_{0}$ serves as the Archimedean order unit for the operator system $C\left(S^{1}\right)^{(n)}$.

The operator system $C\left(S^{1}\right)_{(n)}$ of trigonometric polynomials of degree less than $n$ has a canonical linear basis consisting of functions $\chi_{k}: S^{1} \rightarrow \mathbb{C}$ defined by $\chi_{k}(z)=z^{k}$, for $k=-n+1, \ldots, n-1$. Recall that $\chi_{0}$ is the Archimedean order unit for the operator system $C\left(S^{1}\right)_{(n)}$. 
Let $\left\{\mathfrak{e}_{k} \mid-n+1 \leq k \leq n-1\right\}$ denote the dual basis of the $\left\{\chi_{k} \mid-n+1 \leq k \leq n-1\right\}$; thus, for each $k$,

$$
\mathfrak{e}_{k}[f]=\hat{f}(k),
$$

for every $f \in C\left(S^{1}\right)_{(n)}$. The faithful state $\mathfrak{e}_{0}$ is the designated Archimedean order unit of the operator system dual $\left(C\left(S^{1}\right)_{(n)}\right)^{d}$.

\section{The Connes-van Suijlekom Theorem}

The Connes-van Suijlekom theorem [9, Proposition 4.6] is formulated and proved below in the context of the operator system category. The proof is modeled on the arguments of Connes and van Suijlekom.

Theorem 3.1. $C\left(S^{1}\right)^{(n)} \simeq\left(C\left(S^{1}\right)_{(n)}\right)^{d}$ for every $n \in \mathbb{N}$.

Proof. The case $n=1$ is trivial; therefore, it is assumed that $n \geq 2$.

Consider the linear map $\phi: C\left(S^{1}\right)^{(n)} \rightarrow\left(C\left(S^{1}\right)_{(n)}\right)^{d}$ which takes a Toeplitz matrix $t=\left[\tau_{k-\ell}\right]_{k, \ell=0}^{n-1} \in \mathcal{M}_{n}(\mathbb{C})$ to the linear functional $\varphi_{t}: C\left(S^{1}\right)_{(n)} \rightarrow \mathbb{C}$ defined by

$$
\varphi_{t}(f)=\sum_{k=-n+1}^{n-1} \tau_{-k} a_{k}
$$

where $f(z)=\sum_{k=-n+1}^{n-1} a_{k} z^{k}$. Note that $\phi$ is injective (and hence surjective) and that $\phi$ sends the identity matrix in $C\left(S^{1}\right)^{(n)}$ to the linear functional $f \mapsto \hat{f}(0)$, which we have identified as the Archimedean order unit $\mathfrak{e}_{0}$ of the operator system dual $\left(C\left(S^{1}\right)_{(n)}\right)^{d}$. Therefore, $\phi$ is a unital linear isomorphism, and it remains to show that $\phi$ and $\phi^{-1}$ are completely positive.

To show that $\phi$ is completely positive, fix $p \in \mathbb{N}$ and consider the linear isomorphism $\phi^{[p]}=\phi \otimes \operatorname{id}_{\mathcal{M}_{p}(\mathbb{C})}$. If $T \in C\left(S^{1}\right)^{(n)} \otimes \mathcal{M}_{p}(\mathbb{C})$, then there are matrices $\tau_{\ell} \in \mathcal{M}_{p}(\mathbb{C})$ such that

$$
T=\sum_{\ell=-n+1}^{n-1} r_{\ell} \otimes \tau_{\ell},
$$

which as a matrix is represented as

$$
T=\left[\begin{array}{cccccc}
\tau_{0} & \tau_{-1} & \tau_{-2} & \ldots & \tau_{-n+2} & \tau_{-n+1} \\
\tau_{1} & \tau_{0} & \tau_{-1} & \tau_{-2} & \ldots & \tau_{-n+2} \\
\tau_{2} & \tau_{1} & \tau_{0} & \tau_{-1} & \ddots & \vdots \\
\vdots & \ddots & \ddots & \ddots & \ddots & \tau_{-2} \\
\tau_{n-2} & & \ddots & \ddots & \ddots & \tau_{-1} \\
\tau_{n-1} & \tau_{n-2} & \ldots & \tau_{2} & \tau_{1} & \tau_{0}
\end{array}\right] .
$$

Similarly, $\phi^{[p]}(T)$ is given by

$$
\phi^{[p]}(T)=\sum_{\ell=-n+1}^{n-1} \phi\left(r_{\ell}\right) \otimes \tau_{\ell}=\sum_{\ell=-n+1}^{n-1} \mathfrak{e}_{\ell} \otimes \tau_{\ell} .
$$


To understand the action of $\phi^{[p]}(T)$ on $C\left(S^{1}\right)_{(n)} \otimes \mathcal{M}_{p}(\mathbb{C})$, it is enough to understand the action of $\mathfrak{e}_{\ell} \otimes g$, for some fixed $g \in \mathcal{M}_{p}(\mathbb{C})$. Writing $\mathfrak{e}_{\ell} \otimes g$ as a $p \times p$ matrix of linear functionals on $C\left(S^{1}\right)_{(n)}$, we obtain

$$
\mathfrak{e}_{\ell} \otimes g=\left[\begin{array}{ccc}
g_{11} \mathfrak{e}_{\ell} & \ldots & g_{1 p} \mathfrak{e}_{\ell} \\
\vdots & \ddots & \vdots \\
g_{p 1} \mathfrak{e}_{\ell} & \ldots & g_{p p} \mathfrak{e}_{\ell}
\end{array}\right]
$$

Therefore, if $F \in C\left(S^{1}\right)_{(n)} \otimes \mathcal{M}_{p}(\mathbb{C})$ is given by $F(z)=\sum_{k=-n+1}^{n-1} a_{k} z^{k}$, for some matrices $a_{k} \in \mathcal{M}_{p}(\mathbb{C})$, and if $a_{k}^{i j} \in \mathbb{C}$ denotes the $(i, j)$-entry of $a_{k}$, then

$$
F(z)=\left[\begin{array}{ccc}
\sum_{k=-n+1}^{n-1} a_{k}^{11} z^{k} & \ldots & \sum_{k=-n+1}^{n-1} a_{k}^{1 p} z^{k} \\
\vdots & \ddots & \vdots \\
\sum_{k=-n+1}^{n-1} a_{k}^{p 1} z^{k} & \ldots & \sum_{k=-n+1}^{n-1} a_{k}^{p p} z^{k}
\end{array}\right]
$$

and

$$
\mathfrak{e}_{\ell} \otimes g(F)=\left[\begin{array}{ccc}
g_{11} \mathfrak{e}_{\ell}\left(\sum_{k=-n+1}^{n-1} a_{k}^{11} z^{k}\right) & \ldots & g_{1 p} \mathfrak{e}_{\ell}\left(\sum_{k=-n+1}^{n-1} a_{k}^{1 p} z^{k}\right) \\
\vdots & \ddots & \vdots \\
g_{p 1} \mathfrak{e}_{\ell}\left(\sum_{k=-n+1}^{n-1} a_{k}^{p 1} z^{k}\right) & \ldots & g_{p p} \mathfrak{e}_{\ell}\left(\sum_{k=-n+1}^{n-1} a_{k}^{p p} z^{k}\right)
\end{array}\right],
$$

implying that

$$
\mathfrak{e}_{\ell} \otimes g(F)=\left[\begin{array}{ccc}
g_{11} a_{\ell}^{11} & \ldots & g_{1 p} a_{\ell}^{1 p} \\
\vdots & \ddots & \vdots \\
g_{p 1} a_{\ell}^{p 1} & \ldots & g_{p p} a_{\ell}^{p p}
\end{array}\right]=g \circ a_{\ell},
$$

the Schur-Hadamard product of $g$ with $a_{\ell}$. Therefore, if $T$ is the block Toeplitz matrix in (3.2), then the evaluation of $\phi^{[p]}(T)$ at $F(z)=\sum_{k=-n+1}^{n-1} a_{k} z^{k}$ is given by

$$
\phi^{[p]}(T)[F]=\sum_{k=-n+1}^{n-1} \tau_{-k} \circ a_{k} .
$$

We now show that if $T$ is positive, then $\phi^{[p]}(T)[F]$ is positive for every positive $F \in C\left(S^{1}\right)_{(n)} \otimes \mathcal{M}_{p}(\mathbb{C})$. To this end, assume that the matrix $T$ in (3.2) is positive. Thus, the matrix $\tilde{T}$ whose $(k, \ell)$-entry is $\tau_{k-\ell} \otimes 1_{p}$, where $1_{p}$ denotes the identity matrix of $\mathcal{M}_{p}(\mathbb{C})$, is positive in $\mathcal{M}_{n}\left(\mathcal{M}_{p}(\mathbb{C}) \otimes \mathcal{M}_{p}(\mathbb{C})\right)$. That is, the matrix $\tilde{T}$ is a positive operator on the Hilbert space $\mathcal{H}$ constructed from the direct sum of $n$ copies of $\mathbb{C}^{p} \otimes \mathbb{C}^{p}$. Hence, if $\eta \in \mathcal{H}$ is given by $\eta=\bigoplus_{i=0}^{n-1} \eta_{i}$, where each $\eta_{i} \in \mathbb{C}^{p} \otimes \mathbb{C}^{p}$, then

$$
\begin{aligned}
\langle\tilde{T} \eta, \eta\rangle & =\sum_{i=0}^{n-1} \sum_{k=0}^{n-1}\left\langle\left(\tau_{i-k} \otimes 1_{p}\right) \eta_{k}, \eta_{i}\right\rangle \\
& =\sum_{\ell=-n+1}^{n-1} \sum_{j \in I_{\ell}}\left\langle\left(\tau_{-\ell} \otimes 1_{p}\right) \eta_{\ell+j}, \eta_{j}\right\rangle \\
& \geq 0,
\end{aligned}
$$

where the set $I_{j}$ is given by

$$
I_{j}=\{j \in\{0,1, \ldots, n-1\} \mid \ell+j \geq 0\} .
$$


If $F: S^{1} \rightarrow \mathcal{M}_{p}(\mathbb{C})$ is a positive matrix-valued function of the form $F(z)=$ $\sum_{k=-n+1}^{n-1} a_{k} z^{k}$, for some $a_{k} \in \mathcal{M}_{p}(\mathbb{C})$, then, by the operator-valued Riesz-Fejér theorem [10, 18], there exist $b_{0}, \ldots, b_{n-1} \in \mathcal{M}_{p}(\mathbb{C})$ such that $F(z)=H(z)^{*} H(z)$ for all $z \in S^{1}$, where

$$
H(z)=\sum_{k=0}^{n-1} b_{k} z^{k}
$$

In computing the product $H(z)^{*} H(z)$, we obtain

$$
F(z)=\sum_{\ell=-n+1}^{n-1}\left(\sum_{j \in I_{\ell}} b_{j}^{*} b_{\ell+j}\right) z^{\ell},
$$

where $I_{j}$ is the set in (3.4). Hence,

$$
a_{\ell}=\sum_{j \in I_{\ell}} b_{j}^{*} b_{\ell+j}
$$

for each $\ell \in\{-n+1, \ldots, n-1\}$.

Consider the element $q=\sum_{\ell=-n+1}^{n-1} \tau_{-\ell} \otimes a_{\ell}$ in $\mathcal{M}_{p}(\mathbb{C}) \otimes \mathcal{M}_{p}(\mathbb{C})$, and select any vector $\xi \in \mathbb{C}^{p} \otimes \mathbb{C}^{p}$. For each $i \in\{0,1, \ldots, n-1\}$, set $\eta_{i}=\left(1_{p} \otimes b_{i}\right) \xi$ and $\eta=\bigoplus_{i=0}^{n-1} \eta_{i} \in \mathcal{H}$. Using

$$
\begin{aligned}
q & =\sum_{\ell=-n+1}^{n-1} \tau_{-\ell} \otimes\left(\sum_{j \in I_{\ell}} b_{j}^{*} b_{\ell+j}\right) \\
& =\sum_{\ell=-n+1}^{n-1} \sum_{j \in I_{\ell}}\left(1_{p} \otimes b_{j}\right)^{*}\left(\tau_{-\ell} \otimes 1_{p}\right)\left(1_{p} \otimes b_{\ell+j}\right),
\end{aligned}
$$

we deduce that

$$
\begin{aligned}
\langle q \xi, \xi\rangle & =\sum_{\ell=-n+1}^{n-1} \sum_{j \in I_{\ell}}\left\langle\left(\tau_{-\ell} \otimes 1_{p}\right)\left(1_{p} \otimes b_{\ell+j}\right) \xi,\left(1_{p} \otimes b_{j}\right) \xi\right\rangle \\
& =\sum_{\ell=-n+1}^{n-1} \sum_{j \in I_{\ell}}\left\langle\left(\tau_{-\ell} \otimes 1_{p}\right) \eta_{\ell+j}, \eta_{j}\right\rangle \\
& =\langle\tilde{T} \eta, \eta\rangle \\
& \geq 0 .
\end{aligned}
$$

Hence, $q$ is a positive element of $\mathcal{M}_{p}(\mathbb{C}) \otimes \mathcal{M}_{p}(\mathbb{C})$.

Denote by $v$ the Schur-Hadamard isometry $v: \mathbb{C}^{p} \rightarrow \mathbb{C}^{p} \otimes \mathbb{C}^{p}$ that implements Schur-Hadamard multiplication of matrices $x$ and $y$ via $x \circ y=v^{*}(x \otimes y) v$. Then, because $q$ is positive, so is

$$
\sum_{k=-n+1}^{n-1} \tau_{-k} \circ a_{k}=\sum_{k=-n+1}^{n-1} v^{*}\left(\tau_{-k} \otimes a_{k}\right) v=v^{*} q v,
$$

which proves that $\phi^{[p]}$ is a positive linear map. Hence, $\phi$ is completely positive.

Turning now to the proof that $\phi^{-1}$ is completely positive, we begin by showing $\phi^{-1}$ is positive. To this end, let $\psi$ be a pure state on $C\left(S^{1}\right)_{(n)}$, and consider weak ${ }^{*}$-closed convex set $\mathfrak{S}_{\psi}$ of all states on $C\left(S^{1}\right)$ that extend $\psi$. By the KreinMilman theorem $\mathfrak{S}_{\psi}$ has an extreme point $\Psi$, and this extreme point is necessarily an extreme point of the state space of the abelian $\mathrm{C}^{*}$-algebra $C\left(S^{1}\right)$; hence $\Psi$ is a 
point evaluation at some point $\lambda \in S^{1}$, implying that $\psi(f)=\sum_{k=-n+1}^{n-1} a_{k} \lambda^{k}$, for all $f(z)=\sum_{k=-n+1}^{n-1} a_{k} z^{k}$. Let $\Lambda$ be the positive Toeplitz matrix given by

$$
\Lambda=\left[\begin{array}{lllll}
1 & \lambda & \lambda^{2} & \ldots & \lambda^{n-1}
\end{array}\right]^{*}\left[\begin{array}{lllll}
1 & \lambda & \lambda^{2} & \ldots & \lambda^{n-1}
\end{array}\right]
$$

and note that $\phi(\Lambda)=\psi$. Hence, $\phi^{-1}(\psi)$ is positive and, therefore, so is $\phi^{-1}(\varphi)$, for every linear functional $\varphi$ that is a limit of positive scalar multiples of convex combinations of pure states on $C\left(S^{1}\right)_{(n)}$. This proves that $\phi^{-1}$ is positive.

Because $\phi^{-1}$ is positive linear map of $\left(C\left(S^{1}\right)_{(n)}\right)^{d}$ into $C\left(S^{1}\right)^{(n)}$, its adjoint $\left(\phi^{-1}\right)^{d}:\left(C\left(S^{1}\right)^{(n)}\right)^{d} \rightarrow C\left(S^{1}\right)_{(n)}$ is also positive. Furthermore, since the operator system $C\left(S^{1}\right)_{(n)}$ is an operator subsystem of the abelian $\mathrm{C}^{*}$-algebra $C\left(S^{1}\right)$ and because every positive linear map of an operator system into a unital abelian $\mathrm{C}^{*}$ algebra is completely positive by Lemma 2.4, the positive linear map $\left(\phi^{-1}\right)^{d}$ is completely positive. Thus, $\phi^{-1}$ is completely positive.

Hence, $\phi$ is a unital complete order isomorphism.

Corollary 3.2. $C\left(S^{1}\right)_{(n)} \simeq\left(C\left(S^{1}\right)^{(n)}\right)^{d}$, for every $n \in \mathbb{N}$.

Proof. With any finite-dimensional operator system $\mathcal{R}$, the Archimedean order unit $e_{\mathcal{R}}$ serves as an Archimedean order unit for the bidual $\mathcal{R}^{d d}$, implying that the operator system $\mathcal{R}^{d d}$ is unitally completely order isomorphic to $\mathcal{R}$. Hence, in passing to operator system duals, Theorem 3.1 yields the conclusion.

\section{Operator systems of TOeplitz Operators}

The canonical orthonormal basis functions for the Hilbert space $L^{2}\left(S^{1}\right)$ are given by $e_{k}(z)=(2 \pi)^{-1 / 2} z^{k}$, for $k \in \mathbb{Z}$, while the Hardy space $H^{2}\left(S^{1}\right)$ is the subspace of $L^{2}\left(S^{1}\right)$ having orthonormal basis $\left\{e_{k}\right\}_{k \geq 0}$. The projection operator on $L^{2}\left(S^{1}\right)$ with range $H^{2}\left(S^{1}\right)$ is denoted by $P$. The linear map $\pi: C\left(S^{1}\right) \rightarrow \mathcal{B}\left(L^{2}\left(S^{1}\right)\right)$ given by $\pi(f)=M_{f}$, the operator of multiplication by $f$ on the Hilbert space $L^{2}\left(S^{1}\right)$, is an isometric *-representation of $C\left(S^{1}\right)$ on $L^{2}\left(S^{1}\right)$, implying that the linear map $\varphi: C\left(S^{1}\right) \rightarrow \mathcal{B}\left(H^{2}\left(S^{1}\right)\right)$ defined by $\varphi(f)=P M_{f \mid H^{2}\left(S^{1}\right)}$ is unital and completely positive. The operator $\varphi(f)$ is denoted by $T_{f}$, the Toeplitz operator with symbol $f$. In expressing a Toeplitz operator $T_{f}$ as a matrix with respect to the canonical orthonormal basis of $H^{2}\left(S^{1}\right)$, the result is an infinite Toeplitz matrix whose $(\ell, j)$ entry of the matrix is given by $\hat{f}(\ell-j)$, for $\ell, j \in\{0,1,2, \ldots\}$.

For each $n \in \mathbb{N}$, let

$$
\mathcal{T}_{(n)}=\left\{T_{f} \mid f \in C\left(S^{1}\right)_{(n)}\right\},
$$

which is an operator system of Toeplitz operators on $H^{2}\left(S^{1}\right)$.

Proposition 4.1. If $\varphi: C\left(S^{1}\right) \rightarrow \mathcal{B}\left(H^{2}\left(S^{1}\right)\right)$ denotes the symbol map $\varphi(f)=T_{f}$, for $f \in C\left(S^{1}\right)$, and if $\varphi_{n}=\varphi_{\mid C\left(S^{1}\right)_{(n)}}$, for every $n \in \mathbb{N}$, then $\varphi_{n}$ is a unital complete order isomorphism of $C\left(S^{1}\right)_{(n)}$ and $\mathcal{T}_{(n)}$.

Proof. The function $\varphi_{n}: C\left(S^{1}\right)_{(n)} \rightarrow \mathcal{B}\left(H^{2}\left(S^{1}\right)\right)$ is a unital completely positive linear map with range $\mathcal{T}_{(n)}$. In considering Fourier coefficients, the linear map $\varphi_{n}$ is clearly a linear isomorphism, and so we aim to prove that $\varphi_{n}^{-1}$ is completely positive.

First note that because $\varphi_{n}^{-1}: \mathcal{T}_{(n)} \rightarrow C\left(S^{1}\right)_{(n)}$ and $C\left(S^{1}\right)_{(n)}$ is an operator subsystem of the abelian $\mathrm{C}^{*}$-algebra $C\left(S^{1}\right)$, the complete positivity of $\varphi_{n}$ is automatic, 
by [25, Theorem 3.9], once it has been shown that $\varphi_{n}$ is positive. To this end, let $T_{f} \in \mathcal{T}_{(n)}$ be a positive Toeplitz operator with symbol $f \in C\left(S^{1}\right)_{(n)}$; thus, the essential spectrum $\operatorname{Sp}_{e}\left(T_{f}\right)$ of $T_{f}$ is a subset of $[0, \infty)$. Because $\operatorname{Sp}_{e}\left(T_{f}\right)=f\left(S^{1}\right)$ (see, for example, $[6, \S 4.6]$ ), the symbol $f$ is a positive element of $C\left(S^{1}\right)$ and, hence, of $C\left(S^{1}\right)_{(n)}$, thereby proving that $\varphi_{n}$ is a positive map.

In passing to duals and applying Theorem 3.1 we obtain another result that seems curious upon first encountering it: the $n \times n$ Toeplitz matrices are dual to the infinite Toeplitz matrices arising from symbols in $C\left(S^{1}\right)_{(n)}$.

Corollary 4.2. $\left(\mathcal{T}_{(n)}\right)^{d} \simeq C\left(S^{1}\right)^{(n)}$.

There is another natural ucp map of interest: the one that maps a Toeplitz operator $T_{f}$ with symbol $f \in C\left(S^{1}\right)_{(n)}$ to the $n \times n$ Toeplitz matrix $t_{f}=\sum_{k=-n+1}^{n-1} \hat{f}(k) r_{k}$. (Equivalently, by Proposition 4.1, the map that sends the infinite Toeplitz matrix $T_{f}$ to its $n \times n$ leading principal submatrix $t_{f}$.) If we denote this map by $\psi_{n}$, then $\psi_{n}: C\left(S^{1}\right)_{(n)} \rightarrow C\left(S^{1}\right)^{(n)}$ is a ucp bijection; however, $\psi_{n}$ is not a complete order isomorphism. For example, in the case $n=2$, the function $f(z)=1+z+z^{-1}$ is not positive on $S^{1}$, even though $f=\psi_{2}^{-1}\left(t_{f}\right)$, where the element $t_{f}=\left[\begin{array}{ll}1 & 1 \\ 1 & 1\end{array}\right]$ of $C\left(S^{1}\right)^{(2)}$ is positive.

\section{Applications to matrix theory}

Theorem 5.1. If $\phi: \mathcal{M}_{n}(\mathbb{C}) \rightarrow \mathcal{B}(\mathcal{H})$ is a positive linear map, and if $\phi_{0}$ denotes the restriction of $\phi$ to the Toeplitz matrices $C\left(S^{1}\right)^{(n)}$, then $\phi_{0}$ is a completely positive linear map.

Proof. The stated assertion is a direct consequence of Lemma 2.5. Theorem 3.1. and the fact that the $\mathrm{C}^{*}$-envelope of $\left(C\left(S^{1}\right)^{(n)}\right)^{d} \simeq C\left(S^{1}\right)_{(n)}$ is abelian.

To illustrate this result above, consider the indecomposable positive linear map $\psi: \mathcal{M}_{3}(\mathbb{C}) \rightarrow \mathcal{M}_{3}(\mathbb{C})$ (known as the Choi map; see [26]) given by

$$
\psi\left(\left[\begin{array}{lll}
\alpha_{11} & \alpha_{12} & \alpha_{13} \\
\alpha_{21} & \alpha_{22} & \alpha_{23} \\
\alpha_{31} & \alpha_{32} & \alpha_{33}
\end{array}\right]\right)=\left[\begin{array}{ccc}
\alpha_{11}+\alpha_{33} & -\alpha_{12} & -\alpha_{13} \\
-\alpha_{21} & \alpha_{22}+\alpha_{11} & -\alpha_{23} \\
-\alpha_{31} & -\alpha_{32} & \alpha_{33}+\alpha_{22}
\end{array}\right] .
$$

Note that, if $x \in \mathcal{M}_{3}(\mathbb{C})$ is a Toeplitz matrix, then $\psi(x)=x \circ g$, the SchurHadamard product of $x$ with the matrix

$$
g=\left[\begin{array}{ccc}
2 & -1 & -1 \\
-1 & 2 & -1 \\
-1 & -1 & 2
\end{array}\right]
$$

By the Geršgorin circle theorem, the eigenvalues of the symmetric matrix $g$ are nonnegative, and so the mapping $\psi$ coincides on $C\left(S^{1}\right)^{(3)}$ with the completely positive map on $\mathcal{M}_{3}(\mathbb{C})$ given by the Schur-Hadamard multiplication of $y \in \mathcal{M}_{3}(\mathbb{C})$ by the positive (semidefinite) matrix $g$.

Theorem 5.1 also admits a version for Toeplitz matrices over nuclear $\mathrm{C}^{*}$-algebras: see Corollary 7.4

Theorem 3.1 also leads to an alternative proof of the following result first established in 13 . concerning the structure of unital linear isometries on the operator system of Toeplitz matrices. 
Proposition 5.2. If $\phi: C\left(S^{1}\right)^{(n)} \rightarrow M_{n}(\mathcal{C})$ is a unital linear isometry, then there exists a unitary $v$ such that $\phi(x)=v^{*} x v$ for every $x \in C\left(S^{1}\right)^{(n)}$.

Proof. Let $W(y)$ denote the numerical range of a matrix $y \in \mathcal{M}_{n}(\mathbb{C})$ :

$$
W(y)=\bigcap_{\alpha, \beta \in \mathbb{C}}\left\{\lambda \in \mathbb{C}|| \alpha \lambda+\beta \mid \leq\left\|\alpha y+\beta 1_{n}\right\|\right\} .
$$

Thus, $W(\phi(x))=W(x)$ for every $x \in C\left(S^{1}\right)^{(n)}$, since $\phi: C\left(S^{1}\right)^{(n)} \rightarrow M_{n}(\mathcal{C})$ is a unital linear isometry. As this is also the case for the lower-triangular nilpotent shift matrix $s \in C\left(S^{1}\right)^{(n)}$, the numerical ranges of the contractions $s$ and $\phi(s)$ coincide; hence, by a theorem of $\mathrm{Wu}\left[27\right.$, there is a unitary $v$ such that then $\phi(s)=v^{*} s v$.

Because an element $x \in C\left(S^{1}\right)^{(n)}$ is positive if and only if the numerical range of $x$ is contained in $[0, \infty)$, the unital linear isometry $\phi$ preserves positivity. Hence, by Theorem [5.1] $\phi$ is a completely positive linear map. Now consider the unital completely positive map $\psi: \mathcal{M}_{n}(\mathbb{C}) \rightarrow \mathcal{M}_{n}(\mathbb{C})$ defined by $\psi(y)=v \phi(y) v^{*}$. The fixed point set $\mathfrak{F}^{\psi}=\left\{y \in \mathcal{M}_{n}(\mathbb{C}) \mid \psi(y)=y\right\}$ contains $s$ and $s^{*}$, and so $\mathfrak{F}^{\psi}$ is an irreducible operator subsystem of $\mathcal{M}_{n}(\mathbb{C})$ such that the restriction of $\psi$ to $\mathfrak{F}^{\psi}$ is the identity map. This implies, by Arveson's Boundary Theorem [5, 7, 11], $\psi(y)=y$ for all $y \in M_{n}(\mathbb{C})$; in particular, $\phi(x)=v^{*} x v$, for every $x \in C\left(S^{1}\right)^{(n)}$.

Let $\mathcal{A}\left(S^{1}\right)^{(n)}$ denote the set of analytic Toeplitz matrices, by which is meant those Toeplitz matrices that are lower-triangular. Note that $\mathcal{A}\left(S^{1}\right)^{(n)}$ is a unital abelian algebra generated by the shift $s$.

Proposition 5.3. If $\phi: \mathcal{A}\left(S^{1}\right)^{(n)} \rightarrow \mathcal{M}_{n}(\mathcal{C})$ is a unital isometry, then there exists a unitary $v$ such that $\phi(a)=v^{*}$ av for every $a \in \mathcal{A}\left(S^{1}\right)^{(n)}$.

Proof. Because the operator system $C\left(S^{1}\right)^{(n)}=\left\{a+b^{*} \mid a, b \in \mathcal{A}\left(S^{1}\right)^{(n)}\right\}$, the mapping $\tilde{\phi}: C\left(S^{1}\right)^{(n)} \rightarrow \mathcal{M}_{n}(\mathcal{C})$ given by

$$
\tilde{\phi}\left(a+b^{*}\right)=\phi(a)+\phi(b)^{*}
$$

is well-defined and determines a unital completely positive linear map 25, Proposition 2.12, 3.5]. Further, because $\|\alpha \tilde{\phi}(s)+\beta 1\|=\|\alpha s+\beta 1\|$ for all $\alpha, \beta \in \mathbb{C}$ (by hypothesis), $\tilde{\phi}(s)$ is a matrix of unit norm with numerical range equal to that of the shift $s$. Applying the proof of Proposition 5.2 to $\tilde{\phi}$, we obtain the stated structure for $\tilde{\phi}$ and, hence, for $\phi$.

\section{Positivity of Block Toeplitz matrices Via tensor Products}

If $\mathcal{S}$ is any operator system, then with respect to the linear basis $\left\{r_{-n+1}, \ldots, r_{n-1}\right\}$ of $C\left(S^{1}\right)^{(n)}$ identified earlier, an arbitrary element $x$ of the algebraic tensor product $C\left(S^{1}\right)^{(n)} \otimes \mathcal{S}$ can be written as

$$
x=\sum_{\ell=n+1}^{n-1} r_{\ell} \otimes s_{\ell},
$$

for some $s_{-n+1}, \ldots, s_{n-1} \in \mathcal{S}$. Hence, the algebraic tensor product $C\left(S^{1}\right)^{(n)} \otimes \mathcal{S}$ is naturally identified with the $*$-closed complex vector space of $n \times n$ Toeplitz 
matrices with entries from $\mathcal{S}$, whereby the element $x$ above is represented as

$$
x=\sum_{\ell=n+1}^{n-1} r_{\ell} \otimes s_{\ell}=\left[\begin{array}{cccccc}
s_{0} & s_{-1} & s_{-2} & \ldots & s_{-n+2} & s_{-n+1} \\
s_{1} & s_{0} & s_{-1} & s_{-2} & \ldots & s_{-n+2} \\
s_{2} & s_{1} & s_{0} & s_{-1} & \ddots & \vdots \\
\vdots & \ddots & \ddots & \ddots & \ddots & s_{-2} \\
s_{n-2} & & \ddots & \ddots & \ddots & s_{-1} \\
s_{n-1} & s_{n-2} & \ldots & s_{2} & s_{1} & s_{0}
\end{array}\right] .
$$

Such elements $x \in C\left(S^{1}\right)^{(n)} \otimes \mathcal{S}$ are, therefore, called block Toeplitz matrices.

The purpose of this section is to consider various ways in which block Toeplitz matrices $x$ can be said to be "positive," particularly in the cases where $\mathcal{S}=\mathcal{M}_{m}(\mathbb{C})$ or $\mathcal{S}=C\left(S^{1}\right)^{(m)}$, as these cases are of special interest in applied mathematics (for example, [19]).

With the case $\mathcal{S}=\mathcal{M}_{m}(\mathbb{C})$, the positivity of block Toeplitz matrices is determined by way of the following theoretical criterion, which is a direct consequence of (the proof of) Theorem 3.1

Proposition 6.1. The following statements are equivalent for a matrix $T \in$ $\mathcal{M}_{n}\left(\mathcal{M}_{m}(\mathbb{C})\right)$ of the form

$$
T=\left[\begin{array}{cccccc}
\tau_{0} & \tau_{-1} & \tau_{-2} & \ldots & \tau_{-n+2} & \tau_{-n+1} \\
\tau_{1} & \tau_{0} & \tau_{-1} & \tau_{-2} & \ldots & \tau_{-n+2} \\
\tau_{2} & \tau_{1} & \tau_{0} & \tau_{-1} & \ddots & \vdots \\
\vdots & \ddots & \ddots & \ddots & \ddots & \tau_{-2} \\
\tau_{n-2} & & \ddots & \ddots & \ddots & \tau_{-1} \\
\tau_{n-1} & \tau_{n-2} & \ldots & \tau_{2} & \tau_{1} & \tau_{0}
\end{array}\right]
$$

where each $\tau_{k} \in \mathcal{M}_{m}(\mathbb{C})$ :

(1) $T$ is positive;

(2) for every positive function $F: S^{1} \rightarrow \mathcal{M}_{m}(\mathbb{C})$ of the form

$$
F(z)=\sum_{k=-n+1}^{n-1} a_{k} z^{k},
$$

for some matrices $a_{k} \in \mathcal{M}_{m}(\mathbb{C})$, the matrix

$$
\sum_{k=-n+1}^{n-1} \tau_{-k} \circ a_{k}
$$

is positive.

6.1. Operator system tensor products. One way to approach the positivity question for block Toeplitz matrices for operator systems $\mathcal{S}$ different from $\mathcal{M}_{m}(\mathbb{C})$ is via tensor products of operator systems.

An operator system tensor product 22$] \mathcal{R} \otimes_{\sigma} \mathcal{S}$ of operator systems $\mathcal{S}$ and $\mathcal{T}$ is an operator system structure $\otimes_{\sigma}$ on the algebraic tensor product $\mathcal{R} \otimes \mathcal{S}$ such that:

(1) $\left(\mathcal{R} \otimes \mathcal{S},\left\{\mathcal{C}_{n}\right\}_{n \in \mathbb{N}}, e_{\mathcal{R}} \otimes e_{\mathcal{S}}\right)$ is an operator system, where $\mathcal{C}_{n} \subseteq \mathcal{M}_{n}(\mathcal{R} \otimes \mathcal{S})$, for each $n$, and $e_{\mathcal{R}}$ and $e_{\mathcal{S}}$ denote the Archimedean order units for $\mathcal{R}$ and $\mathcal{S}$ 
(2) $a \otimes b \in \mathcal{C}_{n m}$, for all $a \in \mathcal{M}_{n}(\mathcal{R})_{+}, b \in \mathcal{M}_{m}(\mathcal{S})_{+}$, and $n, m \in \mathbb{N}$;

(3) for all $n, m \in \mathbb{N}$ and all ucp maps $\phi: \mathcal{R} \rightarrow \mathcal{M}_{n}(\mathbb{C})$ and $\psi: \mathcal{S} \rightarrow \mathcal{M}_{m}(\mathbb{C})$, the linear map $\phi \otimes \psi: \mathcal{R} \otimes_{\sigma} \mathcal{S} \rightarrow \mathcal{M}_{n m}(\mathbb{C})$ is completely positive.

Suppose that $\mathcal{R}_{1} \subseteq \mathcal{S}_{1}$ and $\mathcal{R}_{2} \subseteq \mathcal{S}_{2}$ are inclusions of operator systems. Let $\iota_{j}: \mathcal{R}_{j} \rightarrow \mathcal{S}_{j}$ denote the inclusion maps $\iota_{j}\left(x_{j}\right)=x_{j}$ for $x_{j} \in \mathcal{S}_{j}, j=1,2$, so that the map $\iota_{1} \otimes \iota_{2}: \mathcal{R}_{1} \otimes \mathcal{R}_{2} \rightarrow \mathcal{S}_{1} \otimes \mathcal{S}_{2}$ is a linear inclusion of vector spaces. If $\gamma$ and $\sigma$ are operator system structures on $\mathcal{R}_{1} \otimes \mathcal{R}_{2}$ and $\mathcal{S}_{1} \otimes \mathcal{S}_{2}$ respectively, then we use the notation

$$
\mathcal{R}_{1} \otimes_{\gamma} \mathcal{R}_{2} \subseteq \mathcal{S}_{1} \otimes_{\sigma} \mathcal{S}_{2}
$$

to denote that $\iota_{1} \otimes \iota_{2}: \mathcal{R}_{1} \otimes_{\gamma} \mathcal{R}_{2} \rightarrow \mathcal{S}_{1} \otimes_{\sigma} \mathcal{S}_{2}$ is a (unital) completely positive map. This notation is motivated by the fact that $\iota_{1} \otimes \iota_{2}$ is a completely positive map if and only if, for every $p \in \mathbb{N}$, the cone $\mathcal{M}_{p}\left(\mathcal{R}_{1} \otimes_{\gamma} \mathcal{R}_{2}\right)_{+}$is contained in the cone $\mathcal{M}_{p}\left(\mathcal{S}_{1} \otimes_{\sigma} \mathcal{S}_{2}\right)_{+}$. If, in addition, $\iota_{1} \otimes \iota_{2}$ is a complete order isomorphism onto its range, then we write

$$
\mathcal{R}_{1} \otimes_{\gamma} \mathcal{R}_{2} \subseteq \text { coi } \mathcal{S}_{1} \otimes_{\sigma} \mathcal{S}_{2}
$$

In particular, if $\gamma$ and $\sigma$ are two operator system tensor-product structures on $\mathcal{R} \otimes \mathcal{S}$, then $\mathcal{R} \otimes_{\gamma} \mathcal{S}=\mathcal{R} \otimes_{\sigma} \mathcal{S}$ means that the identity map is a unital complete order isomorphism (equivalently, that the matrix positivity cones for $\mathcal{R} \otimes_{\gamma} \mathcal{S}$ and $\mathcal{R} \otimes_{\sigma} \mathcal{S}$ coincide).

6.2. Minimal and maximal tensor products of Toeplitz matrices. An operator system tensor product is described by indicating what the matrix positivity cones are.

The minimal tensor product $\otimes_{\min }$ is the familiar spatial tensor product in matrix and operator theory: if $\mathcal{R} \subseteq \mathcal{B}(\mathcal{H})$ and $\mathcal{S} \subseteq \mathcal{B}(\mathcal{K})$, where $\mathcal{H}$ and $\mathcal{K}$ are Hilbert spaces, then $\mathcal{R} \otimes_{\min } \mathcal{S}$ is the operator system arising from the natural inclusion of $\mathcal{S} \otimes \mathcal{T}$ into $\mathcal{B}(\mathcal{H} \otimes \mathcal{K})$. In this regard, Proposition 6.1 is a characterisation of the cone $\left(C\left(S^{1}\right)^{(n)} \otimes_{\min } \mathcal{M}_{p}(\mathbb{C})\right)_{+}$

When considering the finite-dimensional operator system $\mathcal{T}_{n}$ of Toeplitz operators $T_{f}$ acting on the Hardy space $H^{2}\left(S^{1}\right)$ with symbols $f \in C\left(S^{1}\right)_{(n)}$, the operator system $\mathcal{T}_{n} \otimes_{\min } \mathcal{T}_{m}$ is the operator system of two-level Toeplitz operators $T_{h}$ acting on the Hardy space $H^{2}\left(S^{1} \times S^{1}\right)$ of the torus $S^{1} \times S^{1}$ using symbols from the set $C\left(S^{1} \times S^{1}\right)_{(n, m)}$ of all continuous functions $h: S^{1} \times S^{1} \rightarrow \mathbb{C}$ of the form

$$
h(z, w)=\sum_{k=-n+1}^{n-1} \sum_{j=-m+1}^{m-1} \tau_{k j} z^{k} w^{j},
$$

for some $\tau_{k j} \in \mathbb{C}$.

The maximal tensor product $\otimes_{\max }$ is the operator system structure on $\mathcal{R} \otimes \mathcal{S}$ obtained through declaring a matrix $x \in \mathcal{M}_{p}(\mathcal{R} \otimes \mathcal{S})$ to be positive if for each $\varepsilon>0$ there are $n, m \in \mathbb{N}, a \in \mathcal{M}_{n}(\mathcal{R})_{+}, b \in \mathcal{M}_{m}(\mathcal{S})_{+}$, and a linear map $\delta: \mathbb{C}^{p} \rightarrow \mathbb{C}^{n} \otimes \mathbb{C}^{m}$ such that

$$
\varepsilon\left(e_{\mathcal{R}} \otimes e_{\mathcal{S}}\right)+x=\delta^{*}(a \otimes b) \delta .
$$

It is more difficult for a matrix to be "max positive" than "min positive." For example, if $x$ is a strictly positive element of $\mathcal{S} \otimes_{\max } \mathcal{R}$, then there exist $N \in \mathbb{N}$, $A=\left[a_{i j}\right]_{i, j} \in \mathcal{M}_{N}(\mathcal{S})_{+}$, and $B=\left[b_{i j}\right]_{i, j} \in \mathcal{M}_{N}(\mathcal{R})_{+}$such that $x=\sum_{i, j} a_{i j} \otimes b_{i j}$. 
Therefore, for $\mathcal{R} \otimes_{\min } \mathcal{S}=\mathcal{R} \otimes_{\max } \mathcal{S}$ to hold, at least one of $\mathcal{S}$ or $\mathcal{R}$ ought to be rich in positive elements and matrices. In general,

$$
\mathcal{R} \otimes_{\max } \mathcal{S} \subseteq_{+} \mathcal{R} \otimes_{\sigma} \mathcal{S} \subseteq_{+} \mathcal{R} \otimes_{\min } \mathcal{S},
$$

for every operator system tensor product structure $\otimes_{\sigma}$ on $\mathcal{R} \otimes \mathcal{S}$.

Theorem 6.2 gives two fundamental results relating the min and max tensor products.

Theorem $6.2([14,22])$. If $\mathcal{R}$ and $\mathcal{S}$ are finite-dimensional operator systems and $\mathcal{T}$ is an arbitrary operator system, then

(1) $\mathcal{T} \otimes_{\min } \mathcal{M}_{m}(\mathbb{C})=\mathcal{T} \otimes_{\max } \mathcal{M}_{m}(\mathbb{C})$, for all $m \in \mathbb{N}$, and

(2) $\left(\mathcal{R} \otimes_{\min } \mathcal{S}\right)^{d} \simeq \mathcal{R}^{d} \otimes_{\max } \mathcal{S}^{d}$.

Concerning the maximal tensor product of Toeplitz matrices and operators, we have:

Proposition 6.3. For all $n, m \in \mathbb{N}$,

$$
C\left(S^{1}\right)^{(n)} \otimes_{\max } C\left(S^{1}\right)^{(m)} \simeq\left(C\left(S^{1}\right)_{(n)} \otimes_{\min } C\left(S^{1}\right)_{(m)}\right)^{d} \simeq\left(\mathcal{T}_{(n)} \otimes_{\min } \mathcal{T}_{(m)}\right)^{d}
$$

and

$$
\mathcal{T}_{(n)} \otimes_{\max } \mathcal{T}_{(m)} \simeq\left(C\left(S^{1}\right)^{(n)}\right)^{d} \otimes_{\max }\left(C\left(S^{1}\right)^{(m)}\right)^{d} \simeq\left(C\left(S^{1}\right)^{(n)} \otimes_{\min } C\left(S^{1}\right)^{(m)}\right)^{d} .
$$

Proof. This is an immediate consequence of Proposition 4.1 and Theorems 3.1 and 6.2 .

Theorem 6.2 demonstrates that

$$
C\left(S^{1}\right)^{(n)} \otimes_{\min } \mathcal{M}_{m}(\mathbb{C})=C\left(S^{1}\right)^{(n)} \otimes_{\max } \mathcal{M}_{m}(\mathbb{C}),
$$

for all $n, m \in \mathbb{N}$. Corollary 6.6 shows that if we change the entries $s_{j}$ of the Toeplitz matrix $x$ in (6.1) from arbitrary $m \times m$ complex matrices to arbitrary $m \times m$ Toeplitz matrices, then the two forms of matrix positivity ( $\min$ and $\max$ ) are distinct. To prepare for the proof, we require Lemma 6.4.

Lemma 6.4. Suppose that $p, m \in \mathbb{N}$ are such that $p$ is prime and $p \geq m$. If $\zeta=e^{2 \pi i / p}$ and if $f \in C\left(S^{1}\right)_{(m)}$, then

$$
\hat{f}(0)=\frac{1}{p} \sum_{k=1}^{p} f\left(\zeta^{k}\right)
$$

Proof. Write $f$ as $f(z)=\sum_{\ell=-m+1}^{m-1} \alpha_{\ell} z^{\ell}$, where $\alpha_{\ell}=\hat{f}(\ell)$ for each $\ell$. Suppose that $\ell \in\{-m+1, \ldots, m-1\}$ is nonzero. Because $p$ is prime, $\zeta^{\ell}$ is a primitive $p$-th root of unity, and so $\left\{\left(\zeta^{\ell}\right)^{k} \mid k=1, \ldots, q\right\}$ is the set of all $p$-th roots of unity. Thus, $\sum_{k=1}^{p}\left(\zeta^{\ell}\right)^{k}=0$. Hence,

$$
\sum_{k=1}^{p} f\left(\zeta^{k}\right)=\sum_{k=1}^{p} \sum_{\ell=-m+1}^{m-1} \alpha_{\ell}\left(\zeta^{k}\right)^{\ell}=\sum_{\ell=-m+1}^{m-1} \alpha_{\ell}\left(\sum_{k=1}^{p}\left(\zeta^{\ell}\right)^{k}\right)=p \alpha_{0},
$$

which completes the proof.

Theorem 6.5 was established in the case $n=m=2$ in [12, Theorem 4.7]; the proof below draws from the proof of that result. 
Theorem 6.5. $C\left(S^{1}\right)_{(n)} \otimes_{\min } C\left(S^{1}\right)_{(m)} \neq C\left(S^{1}\right)_{(n)} \otimes_{\max } C\left(S^{1}\right)_{(m)}$, for all $n, m \geq$ 2 .

Proof. Assume that $C\left(S^{1}\right)_{(n)} \otimes_{\min } C\left(S^{1}\right)_{(m)}=C\left(S^{1}\right)_{(n)} \otimes_{\max } C\left(S^{1}\right)_{(m)}$, contrary to what we aim to prove.

For each $j, n \in \mathbb{N}$ such that $j \leq n$, let $\iota_{j, n}: C\left(S^{1}\right)_{(j)} \rightarrow C\left(S^{1}\right)_{(n)}$ be the canonical inclusion map, and note that this map is completely positive. Because the tensor products $\otimes_{\min }$ and $\otimes_{\max }$ are functorial, the linear map $\iota_{j, n} \otimes \iota_{k, m}$, for $j \leq n$ and $k \leq m$, is a unital completely positive embedding of $C\left(S^{1}\right)_{(j)} \otimes_{\min } C\left(S^{1}\right)_{(k)}$ into $C\left(S^{1}\right)_{(n)} \otimes_{\min } C\left(S^{1}\right)_{(m)}$ and of $C\left(S^{1}\right)_{(j)} \otimes_{\max } C\left(S^{1}\right)_{(k)}$ into $C\left(S^{1}\right)_{(n)} \otimes_{\max } C\left(S^{1}\right)_{(m)}$.

Identify $\mathcal{M}_{2}\left(C\left(S^{1}\right)_{(2)} \otimes_{\min } C\left(S^{1}\right)_{(m)}\right)$ with $C\left(S^{1}\right)_{(2)} \otimes_{\min } \mathcal{M}_{2}\left(C\left(S^{1}\right)_{(m)}\right)$ and let $x \in C\left(S^{1}\right)_{(2)} \otimes_{\min } \mathcal{M}_{2}\left(C\left(S^{1}\right)_{(m)}\right)$ be given by

$$
x=\chi_{0} \otimes b_{0}+\chi_{1} \otimes b_{1}+\chi_{-1} \otimes b_{-1},
$$

where $b_{0}=3 \chi_{0}, b_{1}=\left[\begin{array}{cc}\chi_{1} & 0 \\ 2 \chi_{-1} & -\chi_{1}\end{array}\right]$, and $b_{-1}=b_{1}^{*}$. As shown in [12, Theorem 4.7], this matrix-valued function is a positive element of $C\left(S^{1}\right)_{(2)} \otimes_{\min } \mathcal{M}_{2}\left(C\left(S^{1}\right)_{(m)}\right)$ and the eigenvalues of $x(z, w)$, for $(z, w) \in S^{1} \times S^{1}$, are uniformly bounded below by some $\delta>0$. Therefore, the matrix $x$ is strictly positive in $C\left(S^{1}\right)_{(n)} \otimes_{\min }$ $\mathcal{M}_{2}\left(C\left(S^{1}\right)_{(m)}\right)$, which by hypothesis coincides with $C\left(S^{1}\right)_{(n)} \otimes_{\max } \mathcal{M}_{2}\left(C\left(S^{1}\right)_{(m)}\right)$.

As $x \in C\left(S^{1}\right)_{(n)} \otimes_{\max } \mathcal{M}_{2}\left(C\left(S^{1}\right)_{(m)}\right)$ is strictly positive, there exist, by [12, Lemma 2.7], $N \in \mathbb{N}$ and positive matrices $F=\left[f_{i j}\right]_{i j} \in \mathcal{M}_{N}\left(C\left(S^{1}\right)_{(n)}\right)$ and $G=$ $\left[g_{i j}\right]_{i j} \in \mathcal{M}_{N}\left(C\left(S^{1}\right)_{(m)}\right)$ such that

$$
\chi_{0} \otimes b_{0}+\chi_{1} \otimes b_{1}+\chi_{-1} \otimes b_{-1}=\sum_{i, j=1}^{N} f_{i j} \otimes g_{i j} .
$$

Express each $f_{i j}$ as $f_{i j}=\sum_{\ell=-n+1}^{n-1} \alpha_{\ell}^{(i j)} \chi_{\ell}$ so that the sum above becomes

$$
\chi_{0} \otimes b_{0}+\chi_{1} \otimes b_{1}+\chi_{-1} \otimes b_{-1}=\sum_{\ell=-n+1}^{n-1} \chi_{\ell} \otimes\left(\sum_{i, j=1}^{N} \alpha_{\ell}^{(i j)} g_{i j}\right) .
$$

Thus, by the linear independence of $\left\{\chi_{\ell} \mid \ell=-n+1, \ldots, n-1\right\}$,

$$
\sum_{i, j=1}^{N} \alpha_{\ell}^{(i j)} g_{i j}=0
$$

whenever $|\ell|>1$, and

$$
b_{0}=\sum_{i, j=1}^{N} \alpha_{0}^{(i j)} g_{i j} \text { and } b_{1}=\sum_{i, j=1}^{N} \alpha_{1}^{(i j)} g_{i j}=b_{-1}^{*} .
$$

If $A_{0}=\left[\alpha_{0}^{(i j)}\right]_{i, j=1}^{N}$ and $A_{1}=\left[\alpha_{1}^{(i j)}\right]_{i, j=1}^{N}$, then $A_{0}$ is positive, $A_{1}$ is hermitian, and, by the proof of [12, Lemma 4.6], there exist positive complex matrices $\Gamma_{1}=$ $\left[\gamma_{i, j}^{(1)}\right]_{i, j=1}^{N}$ and $\Gamma_{2}=\left[\gamma_{i, j}^{(2)}\right]_{i, j=1}^{N}$ such that $\Gamma_{1}+\Gamma_{2}=A_{0}$ and

$$
Y=\left[\begin{array}{cc}
h_{1} & b_{1} \\
b_{-1} & h_{2}
\end{array}\right] \in\left(\mathcal{M}_{4}\left(C\left(S^{1}\right)_{(m)}\right)_{+},\right.
$$


where $h_{1}, h_{2} \in \mathcal{M}_{2}\left(C\left(S^{1}\right)_{(m)}\right.$ are given by

$$
h_{k}=\sum_{i, j} \gamma_{i j}^{(k)} g_{i j}, \text { for } k=1,2,
$$

and satisfy $h_{1}+h_{2}=b_{0}$.

As in the proof of [12, Theorem 4.7], $Y$ is unitarily equivalent to a positive matrix-valued function of the form

$$
\tilde{Y}(w)=\left[\begin{array}{cccc}
h_{11}(w) & h_{12} & 1 & 0 \\
h_{21}(w) & h_{22}(w) & 2 & -1 \\
1 & 2 & 3-h_{11}(w) & h_{12}(w) \\
0 & -1 & h_{21}(w) & 3-h_{22}(w)
\end{array}\right],
$$

for some $h_{i j} \in C\left(S^{1}\right)_{(m)}$ in which $\hat{h}_{12}(0)=\hat{h}_{21}(0)=0$. By selecting a prime $p \geq m$ and the primitive $p$-th root of unity $\zeta=e^{2 \pi i / p}$, we have, by Lemma 6.4.

$$
\frac{1}{p} \sum_{k=1}^{p} \tilde{Y}\left(\zeta^{k}\right)=\left[\begin{array}{cccc}
\hat{h}_{11}(0) & 0 & 1 & 0 \\
0 & \hat{h}_{22}(0) & 2 & -1 \\
1 & 2 & 3-\hat{h}_{11}(0) & 0 \\
0 & -1 & 0 & 3-\hat{h}_{22}(0)
\end{array}\right] .
$$

By hypothesis, this matrix above is positive, since $Y$ and $\tilde{Y}$ are positive. However, as shown in the proof of [12, Theorem 4.7], it is not. Therefore, the assumption that $C\left(S^{1}\right)_{(n)} \otimes_{\min } C\left(S^{1}\right)_{(m)}=C\left(S^{1}\right)_{(n)} \otimes_{\max } C\left(S^{1}\right)_{(m)}$ leads to a contradiction.

Corollary 6.6. $C\left(S^{1}\right)^{(n)} \otimes_{\min } C\left(S^{1}\right)^{(m)} \neq C\left(S^{1}\right)^{(n)} \otimes_{\max } C\left(S^{1}\right)^{(m)}$, for all $n, m \geq$ 2 .

Proof. The dual of the equality $C\left(S^{1}\right)^{(n)} \otimes_{\min } C\left(S^{1}\right)^{(m)}=C\left(S^{1}\right)^{(n)} \otimes_{\max } C\left(S^{1}\right)^{(m)}$ is $C\left(S^{1}\right)_{(n)} \otimes_{\min } C\left(S^{1}\right)_{(m)}=C\left(S^{1}\right)_{(n)} \otimes_{\max } C\left(S^{1}\right)_{(m)}$, which is false by Theorem 6.5 .

6.3. The commuting tensor product. The commuting tensor product $\otimes_{\mathrm{c}}$ is the operator system structure on $\mathcal{R} \otimes \mathcal{S}$ obtained by declaring a matrix $X \in \mathcal{M}_{p}(\mathcal{R} \otimes \mathcal{S})$ to be positive if $(\phi \cdot \psi)^{(p)}(X)$ is a positive operator for all pairs of completely positive maps $\phi: \mathcal{R} \rightarrow \mathcal{B}(\mathcal{H})$ and $\psi: \mathcal{S} \rightarrow \mathcal{B}(\mathcal{H})$ with commuting ranges, where $\phi \cdot \psi$ denotes the linear map $\mathcal{R} \otimes \mathcal{S} \rightarrow \mathcal{B}(\mathcal{H})$ defined by $\phi \cdot \psi(x \otimes y)=\phi(x) \psi(y)$, for $x \in \mathcal{R}, y \in \mathcal{S}$.

The relationship of $\otimes_{\mathrm{c}}$ to $\otimes_{\text {min }}$ can be vexing to determine, even for operator systems of low dimension. (The problem of whether $\mathcal{S}_{2} \otimes_{\min } \mathcal{S}_{2}=\mathcal{S}_{2} \otimes_{\mathrm{c}} \mathcal{S}_{2}$, for the operator system $\mathcal{S}_{2}$ generated by the unitary generators of the free group $\mathrm{C}^{*}$-algebra $\mathrm{C}^{*}\left(\mathbb{F}_{2}\right)$, is equivalent to the Connes Embedding Problem [20, Theorem 5.11].) In contrast, the relationship of $\otimes_{\mathrm{c}}$ to $\otimes_{\max }$ can often be discerned, as is the case with the Toeplitz operator system and its dual.

Proposition 6.7. $C\left(S^{1}\right)^{(n)} \otimes_{\mathrm{c}} C\left(S^{1}\right)_{(n)} \neq C\left(S^{1}\right)^{(n)} \otimes_{\max } C\left(S^{1}\right)_{(n)}$, for all $n \geq 2$.

Proof. Via the complete order isomorphism $\left(C\left(S^{1}\right)_{(n)}\right)^{d} \simeq C\left(S^{1}\right)^{(n)}$, the equality of $C\left(S^{1}\right)^{(n)} \otimes_{\mathrm{c}} C\left(S^{1}\right)_{(n)}$ and $C\left(S^{1}\right)^{(n)} \otimes_{\max } C\left(S^{1}\right)_{(n)}$ is possible only if $C\left(S^{1}\right)_{(n)}$ is completely order isomorphic to a $\mathrm{C}^{*}$-algebra $\mathcal{A}$ [20, Proposition 4.3]. As the $\mathrm{C}^{*}$-envelope is invariant under complete order isomorphism, this would imply that $\mathcal{A}=\mathrm{C}_{\mathrm{e}}^{*}(\mathcal{A}) \cong \mathrm{C}_{\mathrm{e}}^{*}\left(C\left(S^{1}\right)_{(n)}\right)=C\left(S^{1}\right)$, yielding a linear isomorphism between the finite-dimensional vector space $C\left(S^{1}\right)_{(n)}$ and the infinite-dimensional vector space $C\left(S^{1}\right)$, which is impossible. 
The matrix ordering of $\mathcal{S} \otimes_{\mathrm{c}} \mathcal{R}$ is achieved through the canonical embedding of the algebraic tensor product $\mathcal{S} \otimes \mathcal{R}$ into the $\mathrm{C}^{*}$-algebra $\mathrm{C}_{\mathrm{u}}^{*}(\mathcal{S}) \otimes \max \mathrm{C}_{\mathrm{u}}^{*}(\mathcal{R})[22$; that is,

$$
\mathcal{S} \otimes_{\mathrm{c}} \mathcal{R} \subseteq \text { coi } \mathrm{C}_{\mathrm{u}}^{*}(\mathcal{S}) \otimes_{\max } \mathrm{C}_{\mathrm{u}}^{*}(\mathcal{R}) .
$$

Even so, the universal $\mathrm{C}^{*}$-algebras of $C\left(S^{1}\right)^{(n)}$ and $C\left(S^{1}\right)_{(n)}$ are not sufficiently tractable (e.g., see Theorem 2.1) to draw additional information from.

\section{The maXimally entangled Toeplitz matrix}

Definition 7.1. If $\otimes_{\sigma}$ is an operator system tensor product structure on $\mathcal{S} \otimes \mathcal{R}$, for operator systems $\mathcal{S}$ and $\mathcal{R}$, then an element $x \in\left(\mathcal{S} \otimes_{\sigma} \mathcal{R}\right)_{+}$is:

(1) $\sigma$-separable, if there exist $k \in \mathbb{N}, s_{1}, \ldots, s_{k} \in \mathcal{S}_{+}$, and $r_{1}, \ldots, r_{k} \in \mathcal{R}_{+}$such that $x=\sum_{j=1}^{k} s_{j} \otimes r_{j} ;$ or

(2) $\sigma$-entangled, if $x$ is not $\sigma$-separable.

In the case where $\otimes_{\sigma}$ is the minimal operator system tensor product $\otimes_{\text {min }}$, then we simply refer to $x \in\left(\mathcal{S} \otimes_{\min } \mathcal{R}\right)_{+}$as being separable or entangled.

A beautiful result of Gurvits shows that positive block Toeplitz matrices with blocks coming from $\mathcal{M}_{m}(\mathbb{C})$ are separable.

Theorem 7.2 (Gurvits). Every positive element of $C\left(S^{1}\right)^{(n)} \otimes_{\min } \mathcal{M}_{m}(\mathbb{C})$ is separable.

Proof. The proof of Gurvits' theorem given in [15, §III] yields the result for the operator system $C\left(S^{1}\right)^{(n)} \otimes_{\min } \mathcal{M}_{m}(\mathbb{C})$, although the result is predominantly cited in the literature as pertaining to the matrix algebra $\mathcal{M}_{n}(\mathbb{C}) \otimes_{\min } \mathcal{M}_{m}(\mathbb{C})$.

Corollary 7.3. If $\mathcal{A}$ is a unital nuclear $C^{*}$-algebra and $x \in\left(C\left(S^{1}\right)^{(n)} \otimes_{\min } \mathcal{A}\right)_{+}$, then for every $\varepsilon>0$ there exists a separable $y \in\left(C\left(S^{1}\right)^{(n)} \otimes_{\min } \mathcal{A}\right)_{+}$such that $\|x-y\|<\varepsilon$.

Proof. As $\mathcal{A}$ is nuclear, there exists nets $\left\{\psi_{\lambda}\right\}_{\lambda}$ and $\left\{\phi_{\lambda}\right\}_{\lambda}$ of completely positive linear maps $\psi_{\lambda}: \mathcal{A} \rightarrow \mathcal{M}_{n_{\lambda}}$ and $\phi_{\lambda}: \mathcal{M}_{n_{\lambda}} \rightarrow \mathcal{A}$ such that $\lim _{\lambda}\left\|\phi_{\lambda} \circ \psi_{\lambda}(a)-a\right\|=0$ for every $a \in \mathcal{A}$. If $\iota_{n}$ denotes the identity map on $C\left(S^{1}\right)^{(n)}$, then by Gurvits' theorem there exist, for each $\lambda$, positive Toeplitz matrices $t_{1}^{\lambda}, \ldots, t_{k_{\lambda}}^{\lambda} \in C\left(S^{1}\right)^{(n)}$ and positive $g_{1}^{\lambda}, \ldots, g_{k_{\lambda}}^{\lambda} \in \mathcal{M}_{n_{\lambda}}(\mathbb{C})$ such that

$$
\left(\iota_{n} \otimes \psi_{\lambda}\right)[x]=\sum_{j=1}^{k_{\lambda}} t_{j}^{\lambda} \otimes g_{j}^{\lambda} .
$$

Thus,

$$
\left(\iota_{n} \otimes \phi_{\lambda}\right) \circ\left(\iota_{n} \otimes \psi_{\lambda}\right)[x]=\sum_{j=1}^{k_{\lambda}} t_{j}^{\lambda} \otimes \phi_{\lambda}\left(g_{j}^{\lambda}\right) .
$$

Because $\lim _{\lambda}\left\|x-\left(\iota_{n} \otimes \phi_{\lambda}\right) \circ\left(\iota_{n} \otimes \psi_{\lambda}\right)[x]\right\|=0$, for each $\varepsilon>0$ there exists a separable $y \in\left(C\left(S^{1}\right)^{(n)} \otimes_{\min } \mathcal{A}\right)_{+}$with $\|x-y\|<\varepsilon$.

The second consequence of Gurvits' separation theorem extends Theorem 5.1 , showing that every positive linear map $\psi$ of a unital nuclear $\mathrm{C}^{*}$-algebra $\mathcal{A}$ is "Toeplitz completely positive." 
Corollary 7.4. If $\mathcal{A}$ is a unital nuclear $C^{*}$-algebra and if $\psi: \mathcal{A} \rightarrow \mathcal{B}(\mathcal{H})$ is a positive linear map, then

$$
\left[\begin{array}{cccc}
\psi\left(a_{0}\right) & \psi\left(a_{-1}\right) & \ldots & \psi\left(a_{-n+1}\right) \\
\psi\left(a_{1}\right) & \ddots & \ddots & \vdots \\
\vdots & \ddots & \ddots & \psi\left(a_{-1}\right) \\
\psi\left(a_{n-1}\right) & \cdots & \psi\left(a_{1}\right) & \psi\left(a_{0}\right)
\end{array}\right]
$$

is a positive operator on $\bigoplus_{1}^{n} \mathcal{H}$, for every positive Toeplitz matrix

$$
\left[\begin{array}{cccc}
a_{0} & a_{-1} & \ldots & a_{-n+1} \\
a_{1} & \ddots & \ddots & \vdots \\
\vdots & \ddots & \ddots & a_{-1} \\
a_{n-1} & \ldots & a_{1} & a_{0}
\end{array}\right]
$$

over $\mathcal{A}$.

Proof. Assume that $x=\left[a_{k-\ell}\right]_{\ell, k=0}^{n-1}$ is a positive Toeplitz matrix over $\mathcal{A}$ and let $\psi^{(n)}=\iota_{n} \otimes \psi$, where $\iota_{n}$ is the identity map on $C\left(S^{1}\right)^{(n)}$. By Corollary 7.3, for each $k \in \mathbb{N}$ there is a positive separable Toeplitz matrix $x_{k} \in C\left(S^{1}\right)^{(n)} \otimes_{\min } \mathcal{A}$ such that $\left\|x-x_{k}\right\|<1 / k$. In writing $x_{k}$ as

$$
x_{k}=\sum_{j=1}^{m_{k}} t_{j}^{(k)} \otimes g_{j}^{(k)},
$$

for some $t_{j}^{(k)} \in\left(C\left(S^{1}\right)^{(n)}\right)_{+}$and $g_{j}^{(k)} \in \mathcal{A}_{+}$, we have

$$
\psi^{(n)}\left(x_{k}\right)=\sum_{j=1}^{m_{k}} t_{j}^{(k)} \otimes \psi\left(g_{j}^{(k)}\right),
$$

which is a positive element of $C\left(S^{1}\right)^{(n)} \otimes_{\min } \mathcal{B}(\mathcal{H})$. As $\psi^{(n)}$ is norm-continuous, $\left\|\psi^{(n)}(x)-\psi^{(n)}\left(x_{k}\right)\right\| \rightarrow 0$ as $k \rightarrow \infty$. Because the spectrum of each $x_{k}$ is nonnegative, the upper semicontinuity of the spectrum as a set-valued function implies that the selfadjoint operator $\psi^{(n)}(x)$ also has nonnegative spectrum. Hence, $\psi^{(n)}(x)$ is a positive operator on $\bigoplus_{1}^{n} \mathcal{H}$.

The maximally entangled state in quantum theory is the density operator

$$
\rho=\sum_{k=1}^{d}\left|\psi_{k}\right\rangle\left\langle\psi_{k}\right|
$$

acting on a $d$-dimensional Hilbert space $\mathcal{H}$, where $\left\langle\psi_{1}\right|, \ldots,\left\langle\psi_{d}\right|$ is a given orthonormal basis of $\mathcal{H}$. As the bra-vectors $\left|\psi_{1}\right\rangle, \ldots,\left|\psi_{d}\right\rangle$ form a dual basis of (the dual space) $\mathcal{H}^{d}$ relative to the basis of ket-vectors $\left\langle\psi_{1}\right|, \ldots,\left\langle\psi_{d}\right|$ of $\mathcal{H}$, it seems natural to say, for a finite-dimensional operator system $\mathcal{S}$ with linear basis $\left\{s_{1}, \ldots, s_{m}\right\}$ and dual basis $\left\{\delta_{1}, \ldots, \delta_{m}\right\}$, that the element

$$
\xi=\sum_{j=1}^{m} s_{j} \otimes \delta_{j}
$$

is maximally entangled in $\mathcal{S} \otimes_{\min } \mathcal{S}^{d}$. This terminology is drawn from [21, Appendix $\mathrm{A}]$. 
To apply this notion in the case where $\mathcal{S}=C\left(S^{1}\right)^{(n)}$, we denote the dual basis of the linear basis $\left\{r_{-n+1}, \ldots, r_{n-1}\right\}$ of $C\left(S^{1}\right)^{(n)}$ by $\left\{\delta_{-n+1}, \ldots, \delta_{n-1}\right\}$. We have the identification $C\left(S^{1}\right)^{(n)} \simeq\left(C\left(S^{1}\right)_{(n)}\right)^{d}$ via the unital complete order isomorphism $\phi: C\left(S^{1}\right)^{(n)} \rightarrow\left(C\left(S^{1}\right)_{(n)}\right)^{d}$ given in Theorem 3.1. The effect of $\phi$ on the canonical linear basis of $C\left(S^{1}\right)^{(n)}$ is

$$
\phi\left(r_{k}\right)=\mathfrak{e}_{-k},
$$

for every $k=-n+1, \ldots, n-1$. Note that the linear basis $\left\{\mathfrak{e}_{-n+1}, \ldots, \mathfrak{e}_{n-1}\right\}$ of $\left(C\left(S^{1}\right)_{(n)}\right)^{d}$ is dual to the linear basis $\left\{\chi_{-n+1}, \ldots, \chi_{n-1}\right\}$ of $C\left(S^{1}\right)_{(n)}$. Therefore, if $\psi$ is the unital complete order isomorphism that implements $\left(C\left(S^{1}\right)^{(n)}\right)^{d} \simeq$ $C\left(S^{1}\right)_{(n)}$, then

$$
\psi\left(\delta_{k}\right)=\chi_{-k},
$$

for each $k=-n+1, \ldots, n-1$. Thus, in equation (7.1) above, we replace each $\delta_{k}$ with $\chi_{-k}$ and arrive at Definition 7.5

Definition 7.5. The element $\xi_{n} \in C\left(S^{1}\right)^{(n)} \otimes C\left(S^{1}\right)_{(n)}$ defined by

$$
\xi_{n}=\sum_{k=-n+1}^{n-1} r_{k} \otimes \chi_{-k}
$$

is called the maximally entangled Toeplitz matrix.

Observe that $\xi_{n}$ can be view as the following function $S^{1} \rightarrow C\left(S^{1}\right)^{(n)}$ :

$$
\xi_{n}(z)=\left[\begin{array}{cccccc}
1 & z & z^{2} & \ldots & z^{n-2} & z^{n-1} \\
z^{-1} & 1 & z & z^{2} & \ldots & z^{n-2} \\
z^{-2} & z^{-1} & 1 & z & \ddots & \vdots \\
\vdots & \ddots & \ddots & \ddots & \ddots & z^{2} \\
z^{2-n} & & \ddots & \ddots & \ddots & z \\
z^{1-n} & z^{2-n} & \ldots & z^{-2} & z^{-1} & 1
\end{array}\right]
$$

In light of the Gurvits separability theorem, it is not obvious a priori that the maximally entangled Toeplitz matrix $\xi_{n} \in C\left(S^{1}\right)^{(n)} \otimes_{\min } C\left(S^{1}\right)_{(n)}$ is entangled in the sense of Definition 7.1 therefore, a proof of entanglement is given in Corollary 7.7. Similarly, when considered as an element of the operator system $C\left(S^{1}\right)^{(n)} \otimes_{\min }$ $C\left(S^{1}\right)$, the Toeplitz matrix $\xi_{n}$ is a norm limit of a sequence of positive separable elements, by Corollary 7.3. nevertheless, $\xi_{n}$ is entangled in this operator system, as shown below.

Proposition 7.6. The matrix $\xi_{n}$ is entangled in $C\left(S^{1}\right)^{(n)} \otimes_{\min } C\left(S^{1}\right)$, if $n \geq 2$.

Proof. Assume, on the contrary, that there are nonzero positive Toeplitz matrices $t_{1}, \ldots, t_{k} \in C\left(S^{1}\right)^{(n)}$ and nonzero positive functions $f_{1}, \ldots, f_{k} \in C\left(S^{1}\right)$ such that

$$
\xi_{n}=\sum_{j=1}^{m} t_{j} \otimes f_{j} .
$$

Considering these elements as matrix-valued functions, we have, for every $z \in S^{1}$,

$$
\xi_{n}(z)=\sum_{j=1}^{m} f_{j}(z) t_{j} .
$$


Fix $z \in S^{1}$. The positive matrix $\xi_{n}(z)$ has rank-1; hence, equation (7.3) shows that every vector in $\mathbb{C}^{n}$ annihilated by $\xi_{n}(z)$ is also annihilated by $f_{j}(z) t_{j}$ for every $j$. In passing to orthogonal complements and making use of the fact that these matrices are selfadjoint, we deduce that the rank of each $f_{j}(z) t_{j}$ is 0 or 1 and that the range of each $f_{j}(z) t_{j}$ is contained in $\xi_{n}(z)$. Hence, there are scalars $\alpha_{j}(z) \in[0,1]$, for $j=1, \ldots, m$, such that

$$
f_{j}(z) t_{j}=\alpha_{j}(z) \xi_{n}(z)
$$

Now allowing $z$ to be arbitrary, we see that equation (7.4) holds for every $z \in S^{1}$.

As there are no $z \in S^{1}$ for which $\xi_{n}(z)$ is diagonal, at least one $t_{j}$ must be nondiagonal. With such a $j$, write $t_{j}=\left[\tau_{k-\ell}^{(j)}\right]_{k, \ell=0}^{n-1}$. As $t_{j}$ is positive and nonzero, its diagonal entry satisfies $\tau_{0}^{(j)}>0$; and because $t_{j}$ is not diagonal, there is a $\ell>0$ such that $\tau_{\ell}^{(j)} \neq 0$. Thus, equation (7.4) yields

$$
f_{j}(z) \tau_{0}^{(j)}=\alpha_{j}(z) \text { and } f_{j}(z) \tau_{\ell}^{(j)}=\alpha_{j}(z) z^{-\ell},
$$

for every $z \in S^{1}$. This equation above shows that $\alpha_{j}(z)$ is continuous in $z$ and

$$
\alpha_{j}(z)=\tau_{0}^{(j)} f_{j}(z)=\tau_{\ell}^{(j)}\left(f_{j}(z) z^{\ell}\right),
$$

for every $z \in S^{1}$. Hence, equation (7.5) implies that the Fourier coefficients of $f_{j}$ and $f_{j} \chi_{\ell}$ agree at every $k \in \mathbb{Z}$, which can happen only if $f_{j}$ is identically zero. Because $f_{j}=0$ contradicts the hypothesis that the functions $f_{1}, \ldots, f_{m} \in C\left(S^{1}\right)$ be nonzero, it must be that $\xi_{n}$ is not separable.

Corollary 7.7. The matrix $\xi_{n}$ is entangled in $C\left(S^{1}\right)^{(n)} \otimes_{\min } C\left(S^{1}\right)_{(n)}$, if $n \geq 2$.

Proof. Since $C\left(S^{1}\right)^{(n)} \otimes_{\min } C\left(S^{1}\right)_{(n)} \subseteq$ coi $C\left(S^{1}\right)^{(n)} \otimes_{\min } C\left(S^{1}\right)$, if $\xi_{n}$ were separable in $C\left(S^{1}\right)^{(n)} \otimes_{\min } C\left(S^{1}\right)_{(n)}$, then it would be separable in $C\left(S^{1}\right)^{(n)} \otimes_{\min } C\left(S^{1}\right)$ as well, in contradiction to Proposition 7.6 .

Besides the notion of entanglement, another property of relevance to elements of convex cones is that of purity.

Definition 7.8. An element $x$ of a convex cone $\mathcal{C}$ is pure if the equation $x=y+z$, for $y, z \in \mathcal{C}$, implies that $z=\lambda x$ and $y=(1-\lambda) x$ for some real number $\lambda \in[0,1]$.

Suppose that $f$ and $g$ are positive continuous functions $S^{1} \rightarrow C\left(S^{1}\right)^{(n)}$ such that $f+g=\xi_{n}$. Select $\lambda \in S^{1}$. The positive Toeplitz matrix $\xi_{n}(\lambda)$ has rank-1, and so it is a pure element of the cone $\mathcal{M}_{n}(\mathbb{C})_{+}$, which implies that it is a pure element of the subcone $\left(C\left(S^{1}\right)^{(n)}\right)_{+}$. Hence, from $f(\lambda)+g(\lambda)=\xi_{n}(\lambda)$, there is a scalar $\alpha(\lambda) \in[0,1]$ such that $f(\lambda)=\alpha(\lambda) \xi_{n}(\lambda)$. Thus, the mapping $z \mapsto \alpha(z)$ is a function $\alpha: S^{1} \rightarrow[0,1]$ in which $\alpha(z)=\frac{\|f(z)\|}{\left\|\xi_{n}(z)\right\|}$ for every $z \in S^{1}$. Hence, $\alpha$ is continuous, and we obtain $f(z)=\alpha(z) \xi_{n}(z)$ for all $z \in S^{1}$. Proposition 7.9 indicates that $\alpha$ is a constant function if the Fourier coefficients of $f$ vanish for all $|k| \geq n$.

Proposition 7.9. The maximally entangled Toeplitz matrix $\xi_{n}$ is pure in the convex cone $\left(C\left(S^{1}\right)^{(n)} \otimes_{\min } C\left(S^{1}\right)_{(n)}\right)_{+}$.

Proof. If $\mathcal{W}$ is a finite-dimensional vector space, then the tensor product $\mathcal{W} \otimes \mathcal{W}^{d}$ is linearly isomorphic to $\mathcal{L}(\mathcal{W})$, the vector space of linear transformations on $\mathcal{W}$. If 
we apply this linear isomorphism to a finite-dimensional operator system $C\left(S^{1}\right)^{(n)}$ and its operator system dual $C\left(S^{1}\right)_{(n)}$, then the cone $\mathcal{C P}\left(C\left(S^{1}\right)^{(n)}\right)$ in $\mathcal{L}\left(C\left(S^{1}\right)_{(n)}\right)$ of completely positive linear maps on $C\left(S^{1}\right)^{(n)}$ determines a cone in $C\left(S^{1}\right)^{(n)} \otimes$ $C\left(S^{1}\right)_{(n)}$ : namely, $\left(C\left(S^{1}\right)^{(n)} \otimes_{\min } C\left(S^{1}\right)_{(n)}\right)_{+}[22, \S 4]$.

The canonical linear isomorphism between $\mathcal{R} \otimes \mathcal{R}^{d}$ and $\mathcal{L}(\mathcal{R})$ is the one that maps elementary tensors $x \otimes \psi \in \mathcal{R} \otimes \mathcal{R}^{d}$ to rank-1 linear transformations $r \mapsto \psi(r) x$, for $r \in \mathcal{R}$. Let $\Gamma$ be the inverse of this linear isomorphism and take $\mathcal{R}=C\left(S^{1}\right)^{(n)}$, thereby obtaining a linear isomorphism in which

$$
\Gamma\left(\mathcal{C P}\left(C\left(S^{1}\right)^{(n)}\right)\right)=\left(C\left(S^{1}\right)^{(n)} \otimes_{\min } C\left(S^{1}\right)_{(n)}\right)_{+} .
$$

Observe that $\phi \in \mathcal{C P}(\mathcal{R})$ is pure in the cone $\mathcal{C P}\left(C\left(S^{1}\right)^{(n)}\right)$ if and only if $\Gamma(\phi)$ is pure in the cone $\left(C\left(S^{1}\right)^{(n)} \otimes_{\min } C\left(S^{1}\right)_{(n)}\right)_{+}$.

Let $\iota: C\left(S^{1}\right)^{(n)} \rightarrow C\left(S^{1}\right)^{(n)}$ be the identity map of $C\left(S^{1}\right)^{(n)}$ and note that $\Gamma(\iota)=\xi_{n}$. Thus, we aim to show that $\iota$ is a pure completely positive linear map. To this end, suppose that $\iota=\vartheta+\omega$, for two completely positive linear maps $\vartheta, \omega: C\left(S^{1}\right)^{(n)} \rightarrow C\left(S^{1}\right)^{(n)}$. Viewing these maps as completely positive linear maps from $C\left(S^{1}\right)^{(n)}$ into $\mathcal{M}_{n}(\mathbb{C})$, they admit completely positive linear extensions $\Theta, \Omega: \mathcal{M}_{n}(\mathbb{C}) \rightarrow \mathcal{M}_{n}(\mathbb{C})$. Hence, $\Delta=\Theta+\Omega$ is a ucp map on $\mathcal{M}_{n}(\mathbb{C})$ such that, for each $x \in C\left(S^{1}\right)^{(n)}, \Delta(x)=\theta(x)+\omega(x)=x$. Therefore, by the Boundary Theorem [5,7, 11], $\Delta$ is the identity map of $\mathcal{M}_{n}(\mathbb{C})$. Because the identity map is an irreducible representation of $\mathcal{M}_{n}(\mathbb{C})$, it is pure in the cone of completely positive linear maps on $\mathcal{M}_{n}(\mathbb{C})$. Hence, for some $\lambda \in[0,1], \Theta=\lambda \Delta$ and $\Omega=(1-\lambda) \Delta$, implying that $\vartheta=\lambda \iota$ and $\omega=(1-\lambda) \iota$.

One final point of interest regarding the maximally entangled Toeplitz matrix: it is universal for all (spatially) positive Toeplitz matrices over $\mathrm{C}^{*}$-algebras.

Theorem 7.10 (Ando). If $\mathcal{A}$ is a unital $C^{*}$-algebra and $x \in\left(C\left(S^{1}\right)^{(n)} \otimes_{\min } \mathcal{A}\right)_{+}$, then there exists a completely positive linear map (possibly non-unital)

$$
\phi: C\left(S^{1}\right)^{(n)} \otimes_{\min } C\left(S^{1}\right)_{(n)} \rightarrow C\left(S^{1}\right)^{(n)} \otimes_{\min } \mathcal{A}
$$

such that $\phi\left(\xi_{n}\right)=x$.

Proof. Write $x \in\left(C\left(S^{1}\right)^{(n)} \otimes_{\min } \mathcal{A}\right)_{+}$as $x=\sum_{k=-n+1}^{n-1} r_{k} \otimes a_{k}$, for some $a_{k} \in \mathcal{A}$. Fix $\varepsilon>0$ and set $y=r_{0} \otimes\left(a_{0}+\varepsilon 1\right)^{-1 / 2}$. Let

$$
\tilde{x}=y x y=\sum_{k=-n+1}^{n-1} r_{k} \otimes b_{k},
$$

where $b_{0}=1 \in \mathcal{A}$ and $b_{k}=\left(a_{0}+\varepsilon 1\right)^{-1 / 2} a_{k}\left(a_{0}+\varepsilon 1\right)^{-1 / 2}$. By [1, Theorem 4] (see also [4, Theorem 6.5]), there is a ucp map $\psi: C\left(S^{1}\right) \rightarrow \mathcal{A}$ such that $\psi\left(z^{k}\right)=b_{k}$, for each $k$. Because the transpose map on Toeplitz matrices is induced by a unitary similarity transformation, we may assume that $\psi$ in fact satisfies $\psi\left(z^{k}\right)=b_{-k}$, for each $k$. Let $\psi_{n}$ denote the restriction of $\psi$ to the operator subsystem $C\left(S^{1}\right)_{(n)}$ of $C\left(S^{1}\right)$ and define $\phi: C\left(S^{1}\right)^{(n)} \otimes_{\min } C\left(S^{1}\right)_{(n)} \rightarrow C\left(S^{1}\right)^{(n)} \otimes_{\min } \mathcal{A}$ by

$$
\phi=\left(y^{-1}\right)^{*}\left(\iota_{n} \otimes \psi_{n}\right) y^{-1},
$$

where $\iota_{n}$ is the identity map on $C\left(S^{1}\right)^{(n)}$. Thus, $\phi$ is completely positive and $\phi\left(\xi_{n}\right)=x$. 


\section{Conclusion}

The identification in the operator system category of the operator system $C\left(S^{1}\right)^{(n)}$ of $n \times n$ Toeplitz matrices with the operator system dual of the space $C\left(S^{1}\right)_{(n)}$ of trigonometric polynomials of degree less than $n$ has a number of striking consequences, including the implications that every positive linear map of the Toeplitz matrices is completely positive and every unital $\mathcal{M}_{n}(\mathbb{C})$-valued linear isometric map of $C\left(S^{1}\right)^{(n)}$ is completely isometric. This identification also allows for a clearer understanding of positivity for block Toeplitz matrices, distinguishing block Toeplitz matrices with blocks that are Toeplitz matrices from block Toeplitz matrices with blocks that are arbitrary complex matrices.

An operator system $\mathcal{S}$ has the double commutant expectation property if, for every unital complete order embedding map $\kappa: \mathcal{S} \rightarrow \mathcal{B}(\mathcal{H})$, there exists a unital completely positive linear map $\phi: \mathcal{B}(\mathcal{H}) \rightarrow(\kappa(\mathcal{S}))^{\prime \prime}$ such that $\phi \circ \kappa=\kappa$. Using the work developed in 23], one can show that, for the Toeplitz operator system $C\left(S^{1}\right)^{(n)}$, the double commutant expectation property is equivalent to the assertion that $C\left(S^{1}\right)^{(n)} \otimes_{\min } \mathcal{B}=C\left(S^{1}\right)^{(n)} \otimes_{\max } \mathcal{B}$ for every unital $\mathrm{C}^{*}$-algebra $\mathcal{B}$. (The equality $C\left(S^{1}\right)^{(n)} \otimes_{\min } \mathcal{B}=C\left(S^{1}\right)^{(n)} \otimes_{\max } \mathcal{B}$ is known to hold for all nuclear $\mathrm{C}^{*}$-algebras $\mathcal{B}$ [22.) The case $n=2$ provides some insight into the general situation. To show that $C\left(S^{1}\right)^{(2)}$ has the double commutant expectation property, it is sufficient to prove that every ucp map $\phi: C\left(S^{1}\right)^{(2)} \rightarrow \mathcal{B}(\mathcal{H})$ has a ucp extension $\tilde{\phi}: \mathcal{M}_{2}(\mathbb{C}) \rightarrow$ $\left(\phi\left(C\left(S^{1}\right)^{(2)}\right)\right)^{\prime \prime}$. By Choi's criterion for complete positivity, the extension $\tilde{\phi}$ must have the property that

$$
\left[\begin{array}{ll}
\tilde{\phi}\left(e_{11}\right) & \tilde{\phi}\left(e_{12}\right) \\
\tilde{\phi}\left(e_{21}\right) & \tilde{\phi}\left(e_{22}\right)
\end{array}\right]=\left[\begin{array}{cc}
\tilde{\phi}\left(e_{11}\right) & \phi\left(r_{-1}\right) \\
\phi\left(r_{1}\right) & \tilde{\phi}\left(e_{22}\right)
\end{array}\right]
$$

is positive. Because $1=\phi\left(r_{0}\right)=\tilde{\phi}\left(e_{11}\right)+\tilde{\phi}\left(e_{22}\right)$, the question becomes: given $\phi\left(r_{1}\right)=b$, then is there a positive operator $a$ and a ucp map $\tilde{\phi}: \mathcal{M}_{2}(\mathbb{C}) \rightarrow$ $\left(\phi\left(C\left(S^{1}\right)^{(2)}\right)\right)^{\prime \prime}$ such that $a=\tilde{\phi}\left(e_{11}\right), \tilde{\phi}\left(e_{22}\right)=1-a$, and $b=\tilde{\phi}\left(r_{1}\right)$ ? Ando's theorem [2, 4, 5] gives an affirmative answer. Indeed, since the numerical radius of the matrix $r_{1}$ is $1 / 2$, the numerical radius of $\phi\left(r_{1}\right)$ is at most $1 / 2$; hence, by Ando's theorem, there does indeed exist a positive operator $a$ such that the map $\tilde{\phi}: \mathcal{M}_{2}(\mathbb{C}) \rightarrow \mathcal{B}(\mathcal{H})$ in which $\tilde{\phi}\left(e_{11}\right)=a, \tilde{\phi}\left(e_{22}\right)=1-a$, and $\tilde{\phi}\left(e_{21}\right)=\phi\left(r_{1}\right)$ is completely positive. Some additional arguments can be made (see [12, Theorem $5.4])$ to show that $a$ is in the von Neumann algebra $\left(\phi\left(C\left(S^{1}\right)^{(2)}\right)\right)^{\prime \prime}$, thereby proving that $C\left(S^{1}\right)^{(2)}$ has the double commutant expectation property. For $n>2$, a rather sophisticated version of Ando's numerical radius theorem in several variables would need to hold for $C\left(S^{1}\right)^{(n)}$ to exhibit the double commutant expectation property.

The geometry of the positive cone of $C\left(S^{1}\right)^{(n)}$ is analysed in considerable detail in [9] and further reflection upon the results in that work may reveal previously unobserved phenomena concerning positive Toeplitz matrices.

\section{ACKNOWLEDGEMEnTS}

I am grateful to Mizanur Rahaman for drawing my attention to the works of Gurvits [15] and Ando [3] on the separability of positive Toeplitz matrices, and to the referee for a careful review of the manuscript. 


\section{REFERENCES}

[1] T. Andô, Truncated moment problems for operators, Acta Sci. Math. (Szeged) 31 (1970), 319-334. MR290157

[2] T. Ando, Structure of operators with numerical radius one, Acta Sci. Math. (Szeged) 34 (1973), 11-15. MR318920

[3] T. Ando, Cones and norms in the tensor product of matrix spaces, Linear Algebra Appl. 379 (2004), 3-41, DOI 10.1016/j.laa.2003.06.005. Tenth Conference of the International Linear Algebra Society. MR2039295

[4] Martín Argerami, The matricial range of $E_{21}$, Expo. Math. 37 (2019), no. 1, 48-83, DOI 10.1016/j.exmath.2017.12.002. MR3964216

[5] William Arveson, Subalgebras of $C^{*}$-algebras. II, Acta Math. 128 (1972), no. 3-4, 271-308, DOI 10.1007/BF02392166. MR394232

[6] William Arveson, A short course on spectral theory, Graduate Texts in Mathematics, vol. 209, Springer-Verlag, New York, 2002, DOI 10.1007/b97227. MR.1865513

[7] William Arveson, The noncommutative Choquet boundary, J. Amer. Math. Soc. 21 (2008), no. 4, 1065-1084, DOI 10.1090/S0894-0347-07-00570-X. MR2425180

[8] Man Duen Choi and Edward G. Effros, Injectivity and operator spaces, J. Functional Analysis 24 (1977), no. 2, 156-209, DOI 10.1016/0022-1236(77)90052-0. MR0430809

[9] Alain Connes and Walter D. van Suijlekom, Spectral truncations in noncommutative geometry and operator systems, Comm. Math. Phys. 383 (2021), no. 3, 2021-2067, DOI 10.1007/s00220-020-03825-x. MR4244265

[10] Michael A. Dritschel, On factorization of trigonometric polynomials, Integral Equations Operator Theory 49 (2004), no. 1, 11-42, DOI 10.1007/s00020-002-1198-4. MR2057766

[11] Douglas Farenick, Arveson's criterion for unitary similarity, Linear Algebra Appl. 435 (2011), no. 4, 769-777, DOI 10.1016/j.laa.2011.01.039. MR2807232

[12] Douglas Farenick, Ali S. Kavruk, Vern I. Paulsen, and Ivan G. Todorov, Operator systems from discrete groups, Comm. Math. Phys. 329 (2014), no. 1, 207-238, DOI 10.1007/s00220014-2037-6. MR.3207002

[13] Douglas Farenick, Mitja Mastnak, and Alexey I. Popov, Isometries of the Toeplitz matrix algebra, J. Math. Anal. Appl. 434 (2016), no. 2, 1612-1632, DOI 10.1016/j.jmaa.2015.09.057. MR3415742

[14] Douglas Farenick and Vern I. Paulsen, Operator system quotients of matrix algebras and their tensor products, Math. Scand. 111 (2012), no. 2, 210-243, DOI 10.7146/math.scand.a-15225. MR3023524

[15] Leonid Gurvits and Howard Burnam, Largest separable balls around the maximally mixed bipartite quantum states, Phys. Rev. A 66 (2002), 062311.

[16] Masamichi Hamana, Injective envelopes of operator systems, Publ. Res. Inst. Math. Sci. 15 (1979), no. 3, 773-785, DOI 10.2977/prims/1195187876. MR566081

[17] Samuel J. Harris and Se-Jin Kim, Crossed products of operator systems, J. Funct. Anal. 276 (2019), no. 7, 2156-2193, DOI 10.1016/j.jfa.2018.11.017. MR 3912803

[18] Henry Helson, Lectures on invariant subspaces, Academic Press, New York-London, 1964. MR.0171178

[19] B. Jeuris and R. Vandebril, The Kähler mean of block-Toeplitz matrices with Toeplitz structured blocks, SIAM J. Matrix Anal. Appl. 37 (2016), no. 3, 1151-1175, DOI 10.1137/15M102112X. MR3543154

[20] Ali Ş. Kavruk, Nuclearity related properties in operator systems, J. Operator Theory 71 (2014), no. 1, 95-156, DOI 10.7900/jot.2011nov16.1977. MR.3173055

[21] A. Samil Kavruk, On a non-commutative analogue of a classical result of Namioka and Phelps, J. Funct. Anal. 269 (2015), no. 10, 3282-3303, DOI 10.1016/j.jfa.2015.09.002. MR.3401618

[22] Ali Kavruk, Vern I. Paulsen, Ivan G. Todorov, and Mark Tomforde, Tensor products of operator systems, J. Funct. Anal. 261 (2011), no. 2, 267-299, DOI 10.1016/j.jfa.2011.03.014. MR2793115

[23] Ali S. Kavruk, Vern I. Paulsen, Ivan G. Todorov, and Mark Tomforde, Quotients, exactness, and nuclearity in the operator system category, Adv. Math. 235 (2013), 321-360, DOI 10.1016/j.aim.2012.05.025. MR.3010061 
[24] Eberhard Kirchberg and Simon Wassermann, $C^{*}$-algebras generated by operator systems, J. Funct. Anal. 155 (1998), no. 2, 324-351, DOI 10.1006/jfan.1997.3226. MR.1624549

[25] Vern Paulsen, Completely bounded maps and operator algebras, Cambridge Studies in Advanced Mathematics, vol. 78, Cambridge University Press, Cambridge, 2002. MR 1976867

[26] Kôtarô Tanahashi and Jun Tomiyama, Indecomposable positive maps in matrix algebras, Canad. Math. Bull. 31 (1988), no. 3, 308-317, DOI 10.4153/CMB-1988-044-4. MR956361

[27] Pei Yuan Wu, A numerical range characterization of Jordan blocks, Linear and Multilinear Algebra 43 (1998), no. 4, 351-361, DOI 10.1080/03081089808818536. MR 1616464

Department of Mathematics \& Statistics, University of Regina, Regina, Saskatchewan S4S 0A2, CANADA

Email address: douglas.farenick@uregina.ca 